\title{
On the Vibration Analysis of Rotating Piezoelectric Functionally Graded Beams Resting on Elastic Foundation with a Higher- Order Theory
}

\author{
Pham Minh Phuc $(\mathbb{D}$ and Vu Nguyen Thanh \\ Faculty of Basic Sciences, University of Transport and Communications, 03 Cau Giay Street-Dong Da, Hanoi, Vietnam \\ Correspondence should be addressed to Pham Minh Phuc; bmclt@utc.edu.vn
}

Received 4 April 2021; Revised 28 May 2021; Accepted 24 November 2021; Published 28 January 2022

Academic Editor: Jose Carlos Páscoa

Copyright (C) 2022 Pham Minh Phuc and Vu Nguyen Thanh. This is an open access article distributed under the Creative Commons Attribution License, which permits unrestricted use, distribution, and reproduction in any medium, provided the original work is properly cited.

\begin{abstract}
This work numerically simulates the natural vibration response of rotating piezoelectric functionally graded (FG) beams resting on two-parameter elastic mediums. This is a common kind of design seen in reality, such as marine engine gas turbine blades, rotating railway bridges, and helicopter rotors, where these components may be thought of as beam models rotating around a fixed axis. For the first time, this study uses the finite element method (FEM) in conjunction with Reddy's theory of high-order shear deformation to model the vibration response of a beam rotating around one fixed axis. The present theory eliminates the necessity for shear correction factors while precisely describing the structure's mechanical response. The piezoelectric layers are firmly connected to the top and bottom surfaces of the beam, while the core layer is composed of the FG material, whose material and physical characteristics are expected to gradually change along the thickness direction of the beam in accordance with a power law function as the thickness of the beam is increased. This study is conducted to determine the influences of the structure's geometric and material characteristics on the beam's free vibration behavior, including the rotational speed, distance between the fixed axis and beam endpoint, thickness of piezoelectric layers, and elastic foundation parameters, among other things. Due to the obvious calculation results, the free vibration response of this structure can be easily seen by readers, which serves as a foundation for its design and use in engineering practice.
\end{abstract}

\section{Introduction}

Functionally graded materials (FGM) are the smart and advanced materials made by Japanese scientists in the 1980s. Because they are formed from two or more different materials, they have full advantages over the component materials. The most common type of FGM is made of ceramic and metal, while beam, plate, and shell structures made of FG material store many features that increase the efficiency of these structures such as high strength, resistance to friction and abrasion, good heat resistance, and being difficult to crack. The study of the mechanical responses of these components is interested in researching the scientific community [1-5]. Besides, to increase the efficiency of use for structures made of FG materials, scientists also consider the addition of piezoelectric layers, where the piezoelectric effect produces special effects that are much more prominent without the piezoelectric layer.

Beams made of FG materials with piezoelectric layers are widely used in technical practice and play a significant role in the field. As a consequence, experts all around the globe have been doing research into FG beam structures, as well as piezoelectric FG beams, with remarkable results. In their study, Zhao et al. [6] used strain gradient theory and Euler-Bernoulli beam theory to investigate the effects of porosity and flexoelectricity on the static bending and free 
vibration of porous axially functional gradient piezoelectric nanobeams with axially functional gradient piezoelectric properties. Nan et al. [7] explored the static bending and free vibration analysis of porous functionally graded piezoelectric nanobeams utilizing the electric enthalpy variation and Hamilton's principle, as well as differential equations for regulating the bending and free vibration. Using an analytical method, Singh and Kumari [8] investigated the free vibration analysis of axially functionally graded beams that were integrated with piezoelectric layers and subjected to arbitrarily supported boundary conditions in a free-falling state. Larkin and colleagues [9] used the modified couple stress theory to investigate the impact of small-scale phenomena on the natural frequencies and power density of macro- to nanoscale functionally graded energy harvesters with beam lengths ranging from $62.5 \mathrm{~mm}$ to $6.25 \mathrm{~m}$. They found that the natural frequencies and power density of the harvesters were affected by small-scale phenomena. A two-node efficient finite element model combining layerwise mechanics was used by Yasin and colleagues [10] to assess the active vibration stabilization of piezoelectric functionally graded beams. A functionally graded porous piezoelectric sandwich nanobeam reinforced by graphene platelets was investigated by Chen et al. [11] using the Euler-Bernoulli beam theory and a differential quadrature technique in order to capture the effect of flexoelectricity on the vibration responses of the nanobeam. Askari and colleagues [12] used the classical, first-order, and higher-order beam theories to analyze the natural frequencies of $\mathrm{FG}$ porous beams combined with transverse and shear piezoelectric layers under electrical conditions of short circuit and open circuit.

The rotor of a helicopter, railway track in the turning position, and other elements may be represented as a beam moving around a fixed axis, as can be seen in the illustration. Additionally, mechanical behavior research on these rotating beam constructs is being carried out, with early results having already been published. By improving the geometry of rotating beams, Gunjal and Dixit [13] investigated the lowest vibration states of rotating beams. Simulation of the rotating beam was carried out using the finite-element method, and optimization of the working process was conducted out using sequential quadratic programming. A rotating nanobeam was explored by Pradhan and Murmu [14] utilizing Eringen's nonlocal elasticity theory as well as a single nonlocal beam element to determine its mechanical response. A method known as differential quadrature was used in this investigation. For their study of the free vibration response of a rotating FG beam, $\mathrm{Li}$ and colleagues [15] utilized a dynamic model that took the influences of stretching and bending into consideration. With the use of Euler-Bernoulli, Timoshenko, and finite element beam theories, Amir and colleagues [16] examined the topic of lead-lag vibration in rotating microbeams utilizing rotating microbeams. [17] explored the influences of fractures on the free vibration behavior of a rotating Bernoulli-Euler beam utilizing a unique numerical methodology that allowed for the straightforward computation of these effects using the transfer matrix method, as developed by Jung-Woo and Jung-Youn. Das [18] used the Ritz approach to compute the mechanical reaction of rotating FG beams based on the Timoshenko beam element theory, while also accounting for the Coriolis acceleration. A rotating cantilever beam exposed to magnetic excitations was investigated by $\mathrm{Xu}$ and colleagues [19] using the Hamilton principle and the Galerkin technique. The flow model and vibration suppression of the cantilever beam were investigated. Alireza and Cai [20] conducted a research of the free vibration response of a spinning rod utilizing Eringen's nonlocal elasticity to propose a solution. Liang et al. [21] investigated the free vibration control of a rotating piezoelectric FG beam under temperature settings by using high-order coupling models. A method for capturing the free vibration response of rotating microbeams composed of multilayered composite components with initial geometrical defects was proposed by Dejin and colleagues [22]. The method was based on a combination of the Timoshenko beam and a remodified couple stress theory, which was modified to account for initial geometrical defects. Researchers Atanasov and Stojanović [23] examined the free vibration of rotating nanobeams utilizing a variety of methods including the Euler beam, the Bernoullinonlocal Eringen's elasticity theories, and the Galerkin technique. A large number of different parameter experiments were carried out. Mai et al. [24] evaluated mechanical behaviors of hybrid sandwich plates experimentally. It has recently been used in studies [25-28] and [29] to examine the mechanical behavior of beam and plate structures when rotated. The authors of the work [30] researched the mechanical response of plates while considering temperature and numerous theories. Scientists have studied the mechanical behavior of plate and beam constructions [31-49].

According to the summary above, no papers exist on the rotating piezoelectric FG beams resting on twoparameter elastic foundations with a higher-order theory. Thus, this paper will use a combination of the FEM and Reddy's third-order shear deformation theory (TSDT) to numerically model the free vibration responses of this kind of structure. The suggested method is straightforward, since there are no shear correction variables, and it is simple to construct the stress and strain relations, while yet accurately modeling the mechanical processes. The computational findings presented in this study will provide a solid foundation for the design and use of rotating beam constructions incorporating piezoelectric layers, such as propeller rotors, gas turbine rotors, and rotating railway bridges.

The remainder of this work is arranged in the following manner. Section 2 offers a finite element model of the rotating piezoelectric FG beam based on Reddy beam theory, which takes into consideration the effects of the twoparameter elastic basis on the beam's rotational characteristics. Section 3 includes instances of verification to demonstrate the suggested approach and mechanical models' correctness. Section 4 contains numerical findings and 
comments. Section 5 summarizes various novel points made in this study.

\section{Finite Element Formulations of the Rotating Piezoelectric FG Beam Based on Reddy High- Order Shear Deformation Theory}

This paper focuses on a FG beam rotating at a constant speed around a fixed axis $\Omega$ (see Figure 1 ). The length $L$, the breadth $b$, and the depth $h$ of the beam are its geometrical dimensions, whereas the distance between the beam's terminus and the fixed axis is $r$. The beam is supported by Pasternak's elastic foundation with two parameters, $k_{w}$ an $\eta \mathrm{d} k_{s}$.

The structure's core layer is composed of metal (designated by $m$ ) and ceramic (designated by $c$ ), with the corresponding material volume ratios of $V_{m}$ and $V_{c}$. According to the following power law function $[1,50-55]$, the material ratio of ceramic and metal changes in the depth direction of the core component:

$$
V_{c}=\left(\frac{z}{h}+\frac{1}{2}\right)^{n} ; V_{m}=1-V_{c}, \quad \text { where } n \geq 0
$$

where $z$ is the thickness coordinate variable associated with $-h / 2 \leq z \leq h / 2$ and $n$ is the volume fraction index and its variation.

With the above-mentioned changing law of the material ratio, Young's modulus $E$, Poisson's ratio $v$, and density $\rho$ are all functions of the power law dismemberment as follows [1]:

$$
\begin{aligned}
& E(z)=E_{m}+\left(E_{c}-E_{m}\right)\left(\frac{1}{2}+\frac{z}{h}\right)^{n}, \\
& \rho(z)=\rho_{m}+\left(\rho_{c}-\rho_{m}\right)\left(\frac{1}{2}+\frac{z}{h}\right)^{n}, \\
& v(z)=v_{m}+\left(v_{c}-v_{m}\right)\left(\frac{1}{2}+\frac{z}{h}\right)^{n} .
\end{aligned}
$$

This study makes use of the TSDT in order to develop finite element formulas for estimating the free vibration response of a rotating piezoelectric FG beam, which are then implemented in numerical simulations. Because of this, the displacement components at any location with coordinates $(x, y, z)$ inside the structure have the following form [1], which is presented as follows:

$$
\left\{\begin{array}{l}
u(x, z)=u_{0}(x, 0)+z \beta_{x}-\frac{4}{3 h^{2}} z^{3}\left(\beta_{x}+\frac{\partial w_{0}}{\partial x}\right) \\
w(x, z)=w_{0}(x, 0)
\end{array}\right.
$$

where $u_{0}(x, 0)$ and $w_{0}(x, 0)$ are the displacements along the beam's midaxis and $u$ and $w$ are the movement components along the $x$ - and $z$-axes at any location using the point $(x, z)$.
The strain field is then expressed as follows:

$$
\begin{gathered}
\varepsilon_{x x}=\frac{\partial u}{\partial x}=\frac{\partial u_{0}}{\partial x}+z \frac{\partial \beta_{x}}{\partial x}+\frac{-4 z^{3}}{3 h^{2}}\left(\frac{\partial \beta_{x}}{\partial x}+\frac{\partial^{2} w_{0}}{\partial x^{2}}\right)=\varepsilon_{0 x}+z \varepsilon_{1 x}+z^{3} \varepsilon_{3 x}, \\
\gamma_{x z}=\beta_{x}+\frac{\partial w_{0}}{\partial x}+z^{2} \frac{-4}{h^{2}}\left(\beta_{x}+\frac{\partial w_{0}}{\partial x}\right)=\gamma_{0 x z}+z^{2} \gamma_{2 x z},
\end{gathered}
$$

in which, the strain components are defined as follows:

$$
\begin{aligned}
\varepsilon_{0 x} & =\frac{\partial u_{0}}{\partial x}, \varepsilon_{1 x}=\frac{\partial \beta_{x}}{\partial x}, \varepsilon_{3 x}=\frac{-4}{3 h^{2}}\left(\frac{\partial \beta_{x}}{\partial x}+\frac{\partial^{2} w_{0}}{\partial x^{2}}\right), \gamma_{0 x z} \\
& =\beta_{x}+\frac{\partial w_{0}}{\partial x}, \gamma_{2 x z}=\frac{-4}{h^{2}}\left(\beta_{x}+\frac{\partial w_{0}}{\partial x}\right) .
\end{aligned}
$$

The normal and shear stress components of the FGM core layer of the beam are calculated based on Hooke's law as follows:

$$
\left\{\begin{array}{l}
\sigma_{x x}^{F}=E(z) \varepsilon_{x x} \\
\tau_{x z}^{F}=\frac{E(z)}{2(1+v(z))} \gamma_{x z}
\end{array}\right.
$$

It is possible to describe the linear piezoelectric coupling between the elastic field and an electric field using the direct and reverse piezoelectric equations for the sensor and actuator layers, and the stress-strain relationship can be stated as follows $[56,57]$ for the sensor and actuator layers:

$$
\left\{\begin{array}{l}
\sigma_{x}^{p}=E_{p} \varepsilon_{x x}-e_{31} E_{v}, \\
\tau_{x z}^{p}=G_{p} \gamma_{x z} \\
D^{p}=e_{31} \varepsilon_{x x}+p_{33} E_{v}
\end{array}\right.
$$

where $E_{p}$ and $v_{p}$ are, respectively, elastic modulus and Poisson's ratio of sensor and actuator layers, $D^{p}$ is the electric displacement component, $e_{31}$ denotes the piezoelectric stress coefficient, and $p_{33}$ is the dielectric coefficient. In this study, the influence of the electric field is considered only in the depth direction of the structure. $E_{v}$ is the electric component, which can be calculated through the change of voltage as [58]

$$
E_{v}=\frac{-\phi}{h_{p}}
$$

where $h_{p}$ is the depth of actuator and sensor layers $\left(h_{p}=h_{a}\right.$, $\left.h_{p}=h_{s}\right)$.

The energy of the piezoelectric FG beam resting on Pasternak's elastic medium is calculatedastic medium is 


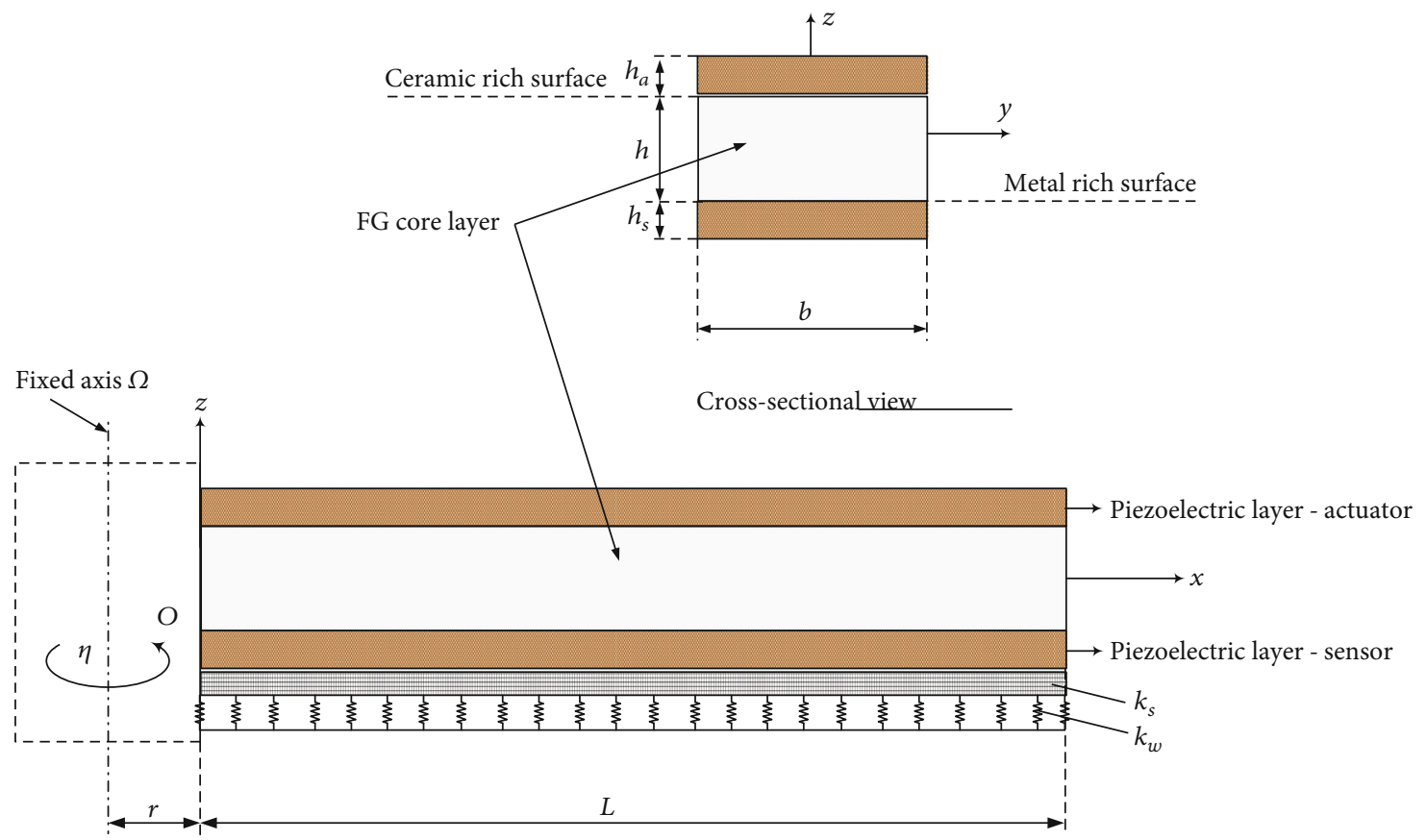

FIGURE 1: The representation of the rotating piezoelectric FG beam resting on Pasternak's elastic medium.

calculated as

$$
\begin{aligned}
& U^{E} d S=\frac{1}{2} \int_{S}\left(\sum_{i} \int_{h_{i}}^{h_{i+1}}\left(\left\{\sigma_{x x}^{F}+\sigma_{x x}^{p}\right\}^{T} \varepsilon_{x x}+\left\{\tau_{x z}^{F}+\tau_{x z}^{p}\right\}^{T} \gamma_{x z}-\left\{D^{p}\right\}^{T} E_{v}\right) d z\right) \\
& =\frac{1}{2} \int_{S}\left(\sum_{i} \int_{h_{i}}^{h_{i+1}}\left(\begin{array}{c}
\varepsilon_{0 x}^{T} E(z) \varepsilon_{0 x}+\varepsilon_{0 x}^{T} z E(z) \varepsilon_{1 x}+\varepsilon_{0 x}^{T} z^{3} E(z) \varepsilon_{3 x} \\
+\varepsilon_{1 x}^{T} E(z) z \varepsilon_{0 x}+\varepsilon_{1 x}^{T} E(z) z^{2} \varepsilon_{1 x}+\varepsilon_{1 x}^{T} E(z) z^{4} \varepsilon_{3 x} \\
+\varepsilon_{3 x}^{T} E(z) z^{3} \varepsilon_{0 x}+\varepsilon_{3 x}^{T} E(z) z^{4} \varepsilon_{1 x}+\varepsilon_{3 x}^{T} E(z) z^{6} \varepsilon_{3 x} \\
+\varepsilon_{0 x}^{T} E_{p} \varepsilon_{0 x}+\varepsilon_{0 x}^{T} z E_{p} \varepsilon_{1 x}+\varepsilon_{0 x}^{T} z^{3} E_{p} \varepsilon_{3 x} \\
+\varepsilon_{1 x}^{T} E_{p} z \varepsilon_{0 x}+\varepsilon_{1 x}^{T} E_{p} z^{2} \varepsilon_{1 x}+\varepsilon_{1 x}^{T} E_{p} z^{4} \varepsilon_{3 x} \\
+\varepsilon_{3 x}^{T} E_{p} z^{3} \varepsilon_{0 x}+\varepsilon_{3 x}^{T} E_{p} z^{4} \varepsilon_{1 x}+\varepsilon_{3 x}^{T} E_{p} z^{6} \varepsilon_{3 x}
\end{array}\right) d z\right) d S \\
& +\frac{1}{2} \int_{S}\left(\sum_{i} \int_{h_{i}}^{h_{i+1}}\left(\begin{array}{c}
-e_{31} E_{v} \varepsilon_{0 x}-e_{31} E_{v} z \varepsilon_{1 x}-e_{31} E_{v} z^{3} \varepsilon_{3 x} \\
-\varepsilon_{0 x}^{T} e_{31} E_{v}-\varepsilon_{1 x}^{T} z e_{31} E_{v}-\varepsilon_{3 x}^{T} z^{3} e_{31} E_{v} \\
-E_{v} p_{33} E_{v}
\end{array}\right) d z\right) d S \\
& +\frac{1}{2} \int_{S}\left(\sum_{i} \int_{h_{i}}^{h_{i+1}}\left(\begin{array}{c}
\gamma_{0 x z}^{T}\left(\frac{E(z)}{2(1+v(z))}\right) \gamma_{0 x z}+\gamma_{0 x z}^{T} G_{p} \gamma_{0 x z} \\
+\gamma_{0 x z}^{T} \frac{E(z)}{2(1+v(z))} z^{2} \gamma_{2 x z}+\gamma_{0 x z}^{T} G_{p} z^{2} \gamma_{2 x z} \\
+\gamma_{2 x z}^{T} \frac{E(z)}{2(1+v(z))} z^{2} \gamma_{0 x z}+\gamma_{2 x z}^{T} G_{p} z^{2} \gamma_{0 x z} \\
+\gamma_{2 x z}^{T} \frac{E(z)}{2(1+v(z))} z^{4} \gamma_{2 x z}+\gamma_{2 x z}^{T} G_{p} z^{4} \gamma_{2 x z}
\end{array}\right)\right) d S \\
& +\frac{1}{2} \int_{S}\left(k_{w} w^{2}+k_{s}\left(\frac{\partial w}{\partial x}\right)^{2}\right) d S+b \int_{L} w^{T} P_{L} d x .
\end{aligned}
$$

where $k_{w}$ and $k_{s}$ are the two parameters of the medium, $b$ is the beam's width, and $P_{L}$ is the external force operating on the beam.
The FG beam rotates along an axis $\Omega$; therefore, the potential energy generated by the structure is computed as follows [22, 59]:

$$
U^{R}=\frac{1}{2} \int_{L}\left(F_{\eta}(x)\left(\frac{\partial w}{\partial x}\right)^{2}\right) d x
$$

where centrifugal force $F_{\eta}$ is defined in the following form [22]:

$$
F_{\eta}=b \sum_{i} \int_{h_{i}}^{h_{i+1}}\left(\left(\rho(z)+\rho_{p}\right) \eta^{2}\left[r(L-x)+\frac{1}{2}\left(L^{2}-x^{2}\right)\right]\right) d z .
$$

In this equation, $\rho_{p}$ denotes the mass density of the piezoelectric layers. The beam's kinetic energy is defined as follows:

$$
\begin{aligned}
K= & \frac{1}{2} \int_{S} \sum_{i} \int_{h_{i}}^{h_{i+1}}\left(( \rho ( z ) + \rho _ { p } ) \left(\left(\frac{\partial \dot{u}^{T}}{\partial t}\right)\left(\frac{\partial \dot{u}}{\partial t}\right)\right.\right. \\
& \left.\left.+\left(\frac{\partial \dot{w}^{T}}{\partial t}\right)\left(\frac{\partial \dot{w}}{\partial t}\right)\right) d z\right) d S .
\end{aligned}
$$

It is necessary to use Hamilton's concept to get the natural vibration equation for the piezoelectric FG beam, which may be written as follows:

$$
\delta \int_{t_{1}}^{t_{2}}\left(K-U^{E}-U^{R o}\right) d t=0
$$


The beam is divided into two-node elements, each with four degrees of freedom:

$$
\psi_{e}=\sum_{i=1}^{2}\left\{\begin{array}{c}
u_{0 i} \\
\beta_{x i} \\
w_{0 i} \\
\left(\frac{\partial w_{0}}{\partial x}\right)_{i}
\end{array}\right\}
$$

Interpolation functions such as Lagrange $\left(N_{i}\right)$ and Hermit $\left(H_{i}\right)$ are used to estimate displacements at any point within the beam using nodal displacements as follows:

$$
\left\{\begin{array}{l}
u_{0}=\sum_{i=1}^{2} N_{i} u_{0 i}=\mathbf{N}_{u} \boldsymbol{\psi}_{e} \\
\beta_{x}=\sum_{i=1}^{2} N_{i} \varphi_{x i}=\mathbf{N}_{\beta} \boldsymbol{\psi}_{e} \\
w_{0}=\sum_{i=1}^{2}\left\{H_{i} w_{0 i}+H_{i+1}\left(\frac{\partial w_{0}}{\partial x}\right)_{i}\right\}=\mathbf{H}_{w} \boldsymbol{\psi}_{e} \\
\frac{\partial w_{0}}{\partial x}=\sum_{i=1}^{2}\left\{\frac{\partial H_{i}}{\partial x} w_{0 i}+\frac{\partial H_{i+1}}{\partial x}\left(\frac{\partial w_{0}}{\partial x}\right)_{i}\right\}_{i}=\mathbf{H}_{w 1 x} \boldsymbol{\psi}_{e} \\
\frac{\partial^{2} w_{0}}{\partial x^{2}}=\sum_{i=1}^{2}\left\{\frac{\partial^{2} H_{i}}{\partial x^{2}} w_{0 i}+\frac{\partial^{2} H_{i+1}}{\partial x^{2}}\left(\frac{\partial w_{0}}{\partial x}\right)_{i}\right\}=\mathbf{H}_{w 2 x} \Psi_{e}
\end{array}\right.
$$

In the matrix form, the following is a more concise version of equation (15):

$$
\mathbf{u}=\left\{\begin{array}{c}
u_{0} \\
\beta_{x} \\
w_{0} \\
\left(\frac{\partial w_{0}}{\partial x}\right)
\end{array}\right\}=\left[\begin{array}{c}
\mathbf{N}_{u} \\
\mathbf{N}_{\beta} \\
\mathbf{H}_{w} \\
\mathbf{H}_{w 1 x}
\end{array}\right] \cdot \psi_{e}=\mathbf{H}_{\psi} \cdot \Psi_{e} \cdot
$$

The strain components are denoted as follows in relation to the nodal displacement vector:

$$
\begin{aligned}
& \left\{\begin{array}{l}
\varepsilon_{0 x}=\frac{\partial u_{0}}{\partial x}=\frac{\partial \mathbf{N}_{u}}{\partial x} \psi_{e}=\mathbf{B}_{0 x} \psi_{e}, \\
\varepsilon_{1 x}=\frac{\partial \beta_{x}}{\partial x}=\frac{\partial \mathbf{N}_{\beta}}{\partial x} \psi_{e}=\mathbf{B}_{1 x} \psi_{e}, \\
\varepsilon_{3 x}=\frac{-4 z^{3}}{3 h^{2}}\left(\frac{\partial \beta_{x}}{\partial x}+\frac{\partial^{2} w_{0}}{\partial x^{2}}\right)=\frac{-4 z^{3}}{3 h^{2}}\left(\frac{\partial \mathbf{N}_{\beta}}{\partial x}+\mathbf{H}_{w 2 x}\right) \psi_{e}=\mathbf{B}_{3 x} \boldsymbol{\psi}_{e},
\end{array}\right. \\
& \left\{\begin{array}{l}
\gamma_{0 x z}=\beta_{x}+\frac{\partial w_{0}}{\partial x}=\left(\mathbf{N}_{\beta}+\mathbf{H}_{w 1 x}\right) \boldsymbol{\psi}_{e}=\mathbf{B}_{0 \gamma} \boldsymbol{\psi}_{e}, \\
\gamma_{2 x z}=\frac{-4}{h^{2}}\left(\beta_{x}+\frac{\partial w_{0}}{\partial x}\right)=\frac{-4}{h^{2}}\left(\mathbf{N}_{\varphi}+\mathbf{H}_{w 1 x}\right) \boldsymbol{\psi}_{e}=\mathbf{B}_{2 \gamma} \boldsymbol{\psi}_{e} .
\end{array}\right.
\end{aligned}
$$

According to the following equation, the electric components of the actuator and sensor layers may be computed via two DOFs of the actuator and sensor components:

$$
\begin{aligned}
& E_{v}^{a}=-\frac{1}{h_{a}} \phi_{a}=B_{\phi}^{a} \phi_{e a} \\
& E_{v}^{s}=-\frac{1}{h_{s}} \phi_{s}=B_{\phi}^{s} \phi_{e s}
\end{aligned}
$$

in which

$$
B_{\phi}^{a}=-\frac{1}{h_{a}} ; B_{\phi}^{s}=-\frac{1}{h_{s}} .
$$

Thus, the energy of an element FG beam is estimated in the following manner:

$$
\begin{aligned}
& U_{e}^{E}=\boldsymbol{\psi}_{e}^{T} \frac{1}{2} \int_{s_{e}}\left\{\sum_{i} \int_{h_{i}}^{h_{i+1}}\left(\begin{array}{c}
\mathbf{B}_{0}^{T} E(z) \mathbf{B}_{0}+\mathbf{B}_{0}^{T} E(z) z \mathbf{B}_{1}+\mathbf{B}_{0}^{T} E(z) z^{3} \mathbf{B}_{3} \\
+\mathbf{B}_{1}^{T} E(z) z \mathbf{B}_{0}+\mathbf{B}_{1}^{T} E(z) z^{2} \mathbf{B}_{1}+\mathbf{B}_{1}^{T} E(z) z^{4} \mathbf{B}_{3} \\
+\mathbf{B}_{3}^{T} E(z) z^{3} \mathbf{B}_{0}+\mathbf{B}_{3}^{T} E(z) z^{4} \mathbf{B}_{1}+\mathbf{B}_{3}^{T} E(z) z^{6} \mathbf{B}_{3} \\
+\mathbf{B}_{0 \gamma}^{T}\left(\frac{E(z)}{2(1+v(z))}\right) \mathbf{B}_{0 \gamma}+\mathbf{B}_{0 \gamma}^{T}\left(\frac{E(z) z^{2}}{2(1+v(z))}\right) \mathbf{B}_{2 \gamma} \\
+\mathbf{B}_{2 \gamma}^{T}\left(\frac{E(z) z^{2}}{2(1+v(z))}\right) \mathbf{B}_{0 \gamma}+\mathbf{B}_{2 \gamma}^{T}\left(\frac{E(z) z^{4}}{2(1+v(z))}\right) \mathbf{B}_{2 \gamma} \\
+k_{w} \mathbf{H}_{w}^{T} \mathbf{H}_{w}+k_{s}\left(\mathbf{H}_{w 1 x}{ }^{T} \mathbf{H}_{w 1 x}\right)
\end{array}\right\} d z\right\} d \psi_{e} \\
& +\boldsymbol{\psi}_{e}^{T} \frac{1}{2} \int_{S_{e}}\left\{\sum_{i} \int_{h_{i}}^{h_{i+1}}\left(\begin{array}{c}
\mathbf{B}_{0}^{T} E_{p} \mathbf{B}_{0}+\mathbf{B}_{0}^{T} E_{p} z \mathbf{B}_{1}+\mathbf{B}_{0}^{T} E_{p} z^{3} \mathbf{B}_{3}+\mathbf{B}_{1}^{T} E_{p} z \mathbf{B}_{0}+\mathbf{B}_{1}^{T} E_{p} z^{2} \mathbf{B}_{1} \\
+\mathbf{B}_{1}^{T} E_{p} z^{4} \mathbf{B}_{3}+\mathbf{B}_{3}^{T} E_{p} z^{3} \mathbf{B}_{0}+\mathbf{B}_{3}^{T} E_{p} z^{4} \mathbf{B}_{1}+\mathbf{B}_{3}^{T} E_{p} z^{6} \mathbf{B}_{3}
\end{array}\right) d z\right\} d S \psi_{e} \\
& +\boldsymbol{\psi}_{e}^{T} \frac{1}{2} \int_{S_{e}}\left\{\sum_{i} \int_{h_{i}}^{h_{i+1}}\left(\begin{array}{c}
\mathbf{B}_{0 \gamma}^{T}\left(G_{p}\right) \mathbf{B}_{0 \gamma}+\mathbf{B}_{0 \gamma}^{T}\left(G_{p} z^{2}\right) \mathbf{B}_{2 \gamma} \\
+\mathbf{B}_{2 \gamma}^{T}\left(G_{p} z^{2}\right) \mathbf{B}_{0 \gamma}+\mathbf{B}_{2 \gamma}^{T}\left(G_{p} z^{4}\right) \mathbf{B}_{2 \gamma}
\end{array}\right) d z\right\} d S \psi_{e}-\boldsymbol{\psi}_{e}^{T} \frac{1}{2} \int_{S_{e}} \\
& \cdot\left(-\mathbf{B}_{0}^{T} e_{31} B_{\phi}^{a}-\mathbf{B}_{1}^{T} z e_{31} B_{\phi}^{a}-\mathbf{B}_{3}^{T} z^{3} e_{31} B_{\phi}^{a}\right) d S \phi_{e a}-\psi_{e}^{T} \frac{1}{2} \int_{S_{e}} \\
& \cdot\left(-\mathbf{B}_{0}^{T} e_{31} B_{\phi}^{s}-\mathbf{B}_{1}^{T} z e_{31} B_{\phi}^{s}-\mathbf{B}_{3}^{T} z^{3} e_{31} B_{\phi}^{s}\right) d S \phi_{e s}-\phi_{e a}^{T} \frac{1}{2} \int_{S_{e}} \\
& \cdot\left(-\left(B_{\phi}^{a}\right)^{T} e_{31} \mathbf{B}_{0}-\left(B_{\phi}^{a}\right)^{T} e_{31} z \mathbf{B}_{1}-\left(B_{\phi}^{a}\right)^{T} e_{31} z^{3} \mathbf{B}_{3}\right) d S \psi_{e}-\phi_{e s}^{T} \frac{1}{2} \int_{S_{e}} \\
& \cdot\left(-\left(B_{\phi}^{s}\right)^{T} e_{31} \mathbf{B}_{0}-\left(B_{\phi}^{s}\right)^{T} e_{31} z \mathbf{B}_{1}-\left(B_{\phi}^{s}\right)^{T} e_{31} z^{3} \mathbf{B}_{3}\right) d S \psi_{e}-\frac{1}{2} \phi_{e a}^{T} \int_{S_{e}} B_{\phi}^{a} P_{33} B_{\phi} d S \phi_{e a} \\
& -\frac{1}{2} \phi_{e s}^{T} \int_{S_{e}} B_{\phi}^{s} p_{33} B_{\phi}^{s} d S \phi_{e s}+\psi_{e}^{T} b \int_{L} \mathbf{H}_{w}^{T} P_{L} d x \text {. }
\end{aligned}
$$

When one rewrites equation (20) in the matrix form, the following equation is obtained:

$$
\begin{aligned}
U_{e}^{E}= & \frac{1}{2} \psi_{e}^{T} \mathbf{K}_{e}^{\psi} \psi_{e}+\frac{1}{2} \psi_{e}^{T} \mathbf{K}_{e}^{\psi \phi_{a}} \phi_{e a}+\frac{1}{2} \psi_{e}^{T} \mathbf{K}_{e}^{\psi \phi_{s}} \phi_{e s} \\
& +\frac{1}{2} \phi_{e a}^{T} \mathbf{K}_{e}^{\phi_{a} \psi} \boldsymbol{\psi}_{e}+\frac{1}{2} \phi_{e s}^{T} \mathbf{K}_{e}^{\phi_{s} \psi} \boldsymbol{\psi}_{e}+\frac{1}{2} \phi_{e a}^{T} \mathbf{K}_{e}^{\phi_{a} \phi_{a}} \phi_{e a} \\
& +\frac{1}{2} \phi_{e s}^{T} \mathbf{K}_{e}^{\phi_{s} \phi_{s}} \phi_{e s}+\boldsymbol{\psi}_{e}^{T} \mathbf{F}_{e} .
\end{aligned}
$$

The energy contained in the centrifugal inertia force is expressed as follows:

$$
U_{e}^{R}=\frac{1}{2} \psi_{e}\left(\int_{L}\left(F_{\eta}(x) \mathbf{H}_{w 1 x}^{T} \mathbf{H}_{w 1 x}\right) d x\right) \boldsymbol{\psi}_{e}=\frac{1}{2} \boldsymbol{\psi}_{e}^{T} \mathbf{K}_{e}^{R} \boldsymbol{\psi}_{e}
$$

The piezoelectric FGM beam element's kinetic energy is 
calculated as follows:

$$
\begin{aligned}
K_{e} & =\frac{1}{2} \int_{S} \sum_{i} \int_{h_{i}}^{h_{i+1}}\left(\dot{\mathbf{u}}^{T}\left(\rho(z)+\rho_{p}\right) \dot{\mathbf{u}}\right) d z d S \\
& =\frac{1}{2} \dot{\psi}_{e}^{T}\left(\int_{S} \sum_{i} \int_{h_{i}}^{h_{i+1}}\left(\rho(z)+\rho_{p}\right)\left(H_{\psi}{ }^{T} H_{\psi}\right) d z d S\right) \dot{\psi}_{e} \\
& =\frac{1}{2} \dot{\psi}_{e}^{T} M_{e} \dot{\psi}_{e}
\end{aligned}
$$

where the element mass matrix is calculated as follows:

$$
\mathbf{M}_{e}=\int \sum_{S} \int_{i}^{h_{i+1}}\left(\rho(z)+\rho_{p}\right)\left(\mathbf{H}_{\psi}{ }^{T} \mathbf{H}_{\psi}\right) d z d S
$$

Substituting equations (21)-(23) into (13), one obtains the formula for obtaining the natural frequencies of the structure as well as the vibration mode as follows:

$$
\left[\begin{array}{ccc}
\mathbf{M}_{e} & 0 & 0 \\
0 & 0 & 0 \\
0 & 0 & 0
\end{array}\right]\left\{\begin{array}{l}
\ddot{\psi}_{e} \\
\ddot{\phi}_{e a} \\
\ddot{\phi}_{e s}
\end{array}\right\}+\left[\begin{array}{ccc}
\mathbf{K}_{e}^{\psi}+\mathbf{K}_{e}^{R} & \mathbf{K}_{e}^{\psi \phi_{a}} & \mathbf{K}_{e}^{\psi \phi_{s}} \\
\mathbf{K}_{e}^{\phi_{a} \psi} & \mathbf{K}_{e}^{\phi_{a} \phi_{a}} & 0 \\
\mathbf{K}_{e}^{\phi_{s} \psi} & 0 & \mathbf{K}_{e}^{\phi_{s} \phi_{s}}
\end{array}\right]\left\{\begin{array}{c}
\psi_{e} \\
\phi_{e a} \\
\phi_{e s}
\end{array}\right\}=\left\{\begin{array}{c}
\mathbf{F}_{e} \\
0 \\
0
\end{array}\right\} .
$$

In the case the external forces are ignored, equation (25) becomes

$$
\left[\begin{array}{ccc}
\mathbf{M}_{e} & 0 & 0 \\
0 & 0 & 0 \\
0 & 0 & 0
\end{array}\right]\left\{\begin{array}{l}
\ddot{\psi}_{e} \\
\ddot{\phi}_{e a} \\
\ddot{\phi}_{e s}
\end{array}\right\}+\left[\begin{array}{ccc}
\mathbf{K}_{e}^{\psi}+\mathbf{K}_{e}^{R} & \mathbf{K}_{e}^{\psi \phi_{a}} & \mathbf{K}_{e}^{\psi \phi_{s}} \\
\mathbf{K}_{e}^{\phi_{a} \psi} & \mathbf{K}_{e}^{\phi_{a} \phi_{a}} & 0 \\
\mathbf{K}_{e}^{\phi_{s} \psi} & 0 & \mathbf{K}_{e}^{\phi_{s} \phi_{s}}
\end{array}\right]\left\{\begin{array}{c}
\psi_{e} \\
\phi_{e a} \\
\phi_{e s}
\end{array}\right\}=\left\{\begin{array}{l}
0 \\
0 \\
0
\end{array}\right\} .
$$

Then, the reduced equation for the degree of displacement freedom of the beam has the following form:

$$
\mathbf{M}_{e} \ddot{\psi}_{e}+\left(\mathbf{K}_{e}^{\psi}+\mathbf{K}_{e}^{R}+\mathbf{K}_{e}^{\psi \phi_{a}}\left(\mathbf{K}_{e}^{\phi_{a} \phi_{a}}\right)^{-1} \mathbf{K}_{e}^{\phi_{a} \psi}+\mathbf{K}_{e}^{\psi \phi_{s}}\left(\mathbf{K}_{e}^{\phi_{s} \phi_{s}}\right)^{-1} \mathbf{K}_{e}^{\phi_{s} \psi}\right) \boldsymbol{\psi}_{e}=0 .
$$

Finally, the equation for determining the natural frequencies and the vibrational shapes of the structure is expressed as

$$
\left\{\sum_{e}\left(\begin{array}{c}
\mathbf{K}_{e}^{\psi}+\mathbf{K}_{e}^{R}+\mathbf{K}_{e}^{\psi \phi_{a}}\left(\mathbf{K}_{e}^{\phi_{a} \phi_{a}}\right)^{-1} \mathbf{K}_{e}^{\phi_{a} \psi} \\
+\mathbf{K}_{e}^{\psi \phi_{s}}\left(\mathbf{K}_{e}^{\phi_{s} \phi_{s}}\right)^{-1} \mathbf{K}_{e}^{\phi_{s} \psi}
\end{array}\right)-\omega^{2} \sum_{e} \mathbf{M}_{e}\right\} \sum_{e} \psi_{e}^{0}=0 .
$$

Thus, by using the TSDT, the element stiffness matrix is calculated without the requirement for a shear correction coefficient, precisely simulating the mechanical response of this beam. Additionally, in the above equation, the component of element stiffness is proportional to the rotational speed of the structure, which affects the beam's mechanical reaction differently than it would be without rotation.
The following are the boundary conditions that are described in this work:

(i) Simply supported (denoted by S):

$$
u_{0}=0, w_{0}=0
$$

(ii) Clamped (denoted by C):

$$
u_{0}=0, w_{0}=0, \psi_{x}=0, \frac{\partial w_{0}}{\partial x}=0
$$

In this work, the S-S and C-C boundaries are utilized in this paper.

\section{Investigation of Verification}

In order to substantiate the hypothesis advanced above, this part presents three instances in which the findings of this study are compared to those of previously published data.

Example 1. At first, this problem compares the data of natural frequencies of the $\mathrm{S}-\mathrm{S}$ piezoelectric $\mathrm{FG}\left(\mathrm{Al} / \mathrm{Al}_{2} \mathrm{O}_{3}\right)$ beam. The length of the beam $L=1 \mathrm{~m}$, thickness $h=L / 10$, and two piezoelectric components have the same depth $h_{a}$ $=h_{s}=h / 5$. Material properties of the FGM core layer: $E_{m}$ $=70 \mathrm{GPa}, \rho_{m}=2700 \mathrm{~kg} / \mathrm{m}^{3}, E_{c}=380 \mathrm{GPa}$, and $\rho_{c}=3800 \mathrm{~kg}$ $/ \mathrm{m}^{3}$. Piezoelectric layers are made from PZT-5H: $E_{p}=$ 127.2 GPa, $G_{p}=23 \mathrm{GPa}, e_{31}=-6.6 \mathrm{C} / \mathrm{m}^{2}, p_{33}=15.1 \mathrm{nF} / \mathrm{m}$, and $\rho_{a}=7500 \mathrm{~kg} / \mathrm{m}^{3}$. In Table 1 , the first two fundamental frequencies of the piezoelectric FG beam calculated using the first-order shear deformation beam theory (FBT), classical beam theory (CBT), sinusoidal shear deformation beam theory (SBT), parabolic shear deformation beam theory (PBT), and exponential shear deformation beam theory (EBT) are presented, in which several various mesh sizes ranging from four to twelve elements are calculated in this study. As the number of pieces in this work rises, it becomes clear that the outcomes of this study are becoming more similar to the published findings. This work makes use of a computer with the following characteristics: a core i5 processor with a processing speed of $1.7 \mathrm{GHz}$ and a RAM speed of 2.7 GHz; the calculation time for each mesh is also provided in Table 1. It has been discovered that as the number of components rises, the time required for computing increases as well.

Example 2. Following that, this example compares the fundamental frequency data of a fully supported $\mathrm{Al} / \mathrm{Al}_{2} \mathrm{O}_{3}$ beam resting on a two-parameter elastic medium. Geometric and material characteristics are length $L$, depth $h, L / h=100$, breadth $b, E_{m}=70 \mathrm{GPa}, \rho_{m}=2702 \mathrm{~kg} / \mathrm{m}^{3}, E_{c}=380 \mathrm{GPa}$, and $\rho_{c}=3960 \mathrm{~kg} / \mathrm{m}^{3}$. The medium's two nondimensional 
TABLE 1: The first two natural frequencies $(\mathrm{Hz})$ of the piezoelectric FGM, S-S $(L=1 \mathrm{~m}, L / h=10)$.

\begin{tabular}{|c|c|c|c|c|c|c|c|c|}
\hline Source & Number of elements & Computational time (s) & $n=0$ & $\begin{array}{c}\text { Mode } 1 \\
n=1\end{array}$ & $n=10$ & $n=0$ & $\begin{array}{c}\text { Mode } 2 \\
n=1\end{array}$ & $n=10$ \\
\hline CBT-Exact [12] & - & - & 435.309 & 374.916 & 349.683 & 1691.218 & 1453.180 & 1353.334 \\
\hline FBT-Exact [12] & - & - & 428.399 & 368.360 & 339.793 & 1600.435 & 1368.370 & 1230.287 \\
\hline PBT-Exact [12] & - & - & 427.996 & 368.151 & 337.747 & 1594.783 & 1365.460 & 1207.247 \\
\hline SBT-Exact [12] & - & - & 427.979 & 368.172 & 337.624 & 1594.517 & 1365.688 & 1205.928 \\
\hline EBT-Exact [12] & - & - & 427.974 & 368.205 & 337.529 & 1594.398 & 1366.072 & 1204.915 \\
\hline \multirow{5}{*}{ This work } & 4 & 0.869 & 424.300 & 368.400 & 345.600 & 1683.70 & 1458.00 & 1264.400 \\
\hline & 6 & 0.874 & 423.100 & 367.400 & 344.700 & 1656.10 & 1434.20 & 1263.200 \\
\hline & 8 & 0.884 & 422.600 & 367.200 & 344.600 & 1648.700 & 1427.500 & 1262.800 \\
\hline & 10 & 0.941 & 422.500 & 367.000 & 344.300 & 1645.900 & 1425.400 & 1262.600 \\
\hline & 12 & 0.995 & 422.500 & 366.900 & 343.500 & 1644.200 & 1423.800 & 1262.400 \\
\hline
\end{tabular}

TABLE 2: The nondimensional frequencies $(\chi)$ of the SS FG beam placed on the two-parameter elastic medium $\left(\widehat{K}_{w}=10, \widehat{K}_{s}=1\right.$, and $L / h$ $=100)$.

\begin{tabular}{|c|c|c|c|c|c|c|c|c|}
\hline & $n$ & Mode & Exact solution [60] & 4 elements & 6 elements & $\begin{array}{l}\text { Present work } \\
8 \text { elements }\end{array}$ & 10 elements & 12 elements \\
\hline \multirow{8}{*}{$\begin{array}{l}\bar{K}_{w}=0 \\
\bar{K}_{s}=0\end{array}$} & \multirow{3}{*}{0} & 1 & 5.483 & 5.505 & 5.491 & 5.484 & 5.482 & 5.480 \\
\hline & & 2 & 21.933 & 22.504 & 22.124 & 22.020 & 21.977 & 21.958 \\
\hline & & 3 & 49.350 & 53.548 & 50.621 & 49.919 & 49.669 & 49.533 \\
\hline & \multirow{3}{*}{1} & 1 & 4.221 & 4.242 & 4.230 & 4.229 & 4.220 & 4.220 \\
\hline & & 2 & 16.884 & 17.335 & 17.044 & 16.966 & 16.931 & 16.919 \\
\hline & & 3 & 37.989 & 41.275 & 39.034 & 38.429 & 38.260 & 38.211 \\
\hline & \multirow{3}{*}{2} & 1 & 3.852 & 3.874 & 3.860 & 3.860 & 3.852 & 3.854 \\
\hline & & 2 & 15.407 & 15.827 & 15.560 & 15.487 & 15.458 & 15.442 \\
\hline \multirow{10}{*}{$\begin{array}{l}\bar{K}_{w}=10 \\
\bar{K}_{s}=1\end{array}$} & & 3 & 34.666 & 37.665 & 35.637 & 35.105 & 34.924 & 34.867 \\
\hline & \multirow{3}{*}{0} & 1 & 6.015 & 6.038 & 6.021 & 6.014 & 6.008 & 6.007 \\
\hline & & 2 & 22.445 & 23.024 & 22.637 & 22.533 & 22.491 & 22.471 \\
\hline & & 3 & 49.858 & 54.077 & 51.158 & 50.441 & 50.164 & 50.056 \\
\hline & \multirow{3}{*}{1} & 1 & 5.008 & 5.030 & 5.014 & 5.015 & 5.001 & 5.000 \\
\hline & & 2 & 17.666 & 18.128 & 17.827 & 17.748 & 17.708 & 17.697 \\
\hline & & 3 & 38.77 & 42.043 & 39.791 & 39.247 & 39.037 & 38.930 \\
\hline & \multirow{3}{*}{2} & 1 & 4.752 & 4.773 & 4.759 & 4.756 & 4.741 & 4.740 \\
\hline & & 2 & 16.316 & 16.748 & 16.472 & 16.395 & 16.360 & 16.353 \\
\hline & & 3 & 35.577 & 38.588 & 36.533 & 36.006 & 35.843 & 35.733 \\
\hline
\end{tabular}

frequencies are computed as follows:

$$
\left\{\begin{array}{l}
\widehat{K}_{w}=\frac{k_{w} L^{4}}{E_{m} I}, \\
\widehat{K}_{s}=\frac{k_{s} L^{2}}{E_{m} I \pi^{2}},
\end{array}\right.
$$

where

$$
I=\frac{b h^{3}}{12} .
$$

The following formula is used to get the beam's nondimensional natural frequency:

$$
\lambda=\omega \frac{L^{2}}{h} \sqrt{\frac{\rho_{m}}{E_{m}}} .
$$

The results in Table 2 are derived from the analytical technique [60] and current work, in which the grid sizes for various unique mesh sizes are estimated. As seen in Examples 1 and 2, accuracy converges at the 10-element mesh size. Because of this, this one will be utilized for all further research. 
TABLE 3: The first four nondimensional fundamental frequencies $(\wp)$ of the C-F rotating beam.

\begin{tabular}{|c|c|c|c|c|c|}
\hline \multirow{2}{*}{ Vibration mode } & \multirow{2}{*}{ Source } & \multicolumn{4}{|c|}{ Speed $\vartheta$} \\
\hline & & $\aleph=0$ & $\aleph=3$ & $\aleph=6$ & $\aleph=12$ \\
\hline \multirow{4}{*}{$\tilde{\omega}_{1}$} & Present work & 3.4787 & 4.7538 & 7.3104 & 13.1661 \\
\hline & Exact $[61]$ & 3.5160 & 4.7973 & 7.3604 & 13.1702 \\
\hline & DMM [62] & 3.5160 & 4.7973 & 7.3604 & - \\
\hline & Isogeometric [22] & 3.5118 & 4.7733 & 7.3276 & - \\
\hline \multirow{4}{*}{$\tilde{\omega}_{2}$} & Present work & 22.1083 & 23.3811 & 26.8126 & 37.5336 \\
\hline & Exact [61] & 22.0345 & 23.3203 & 26.8091 & 37.6031 \\
\hline & DMM [62] & - & - & - & - \\
\hline & Isogeometric [22] & - & - & - & - \\
\hline \multirow{4}{*}{$\tilde{\omega}_{3}$} & Present work & 62.2026 & 63.4748 & 67.1399 & 79.9519 \\
\hline & Exact [61] & 61.6972 & 62.9850 & 66.6840 & 79.6145 \\
\hline & DMM [62] & - & - & - & - \\
\hline & Isogeometric [22] & - & - & - & - \\
\hline \multirow{4}{*}{$\tilde{\omega}_{4}$} & Present work & 123.037 & 124.361 & 128.234 & 142.523 \\
\hline & Exact $[61]$ & 120.902 & 122.236 & 126.140 & 140.534 \\
\hline & DMM [62] & - & - & - & - \\
\hline & Isogeometric [22] & - & - & - & - \\
\hline
\end{tabular}

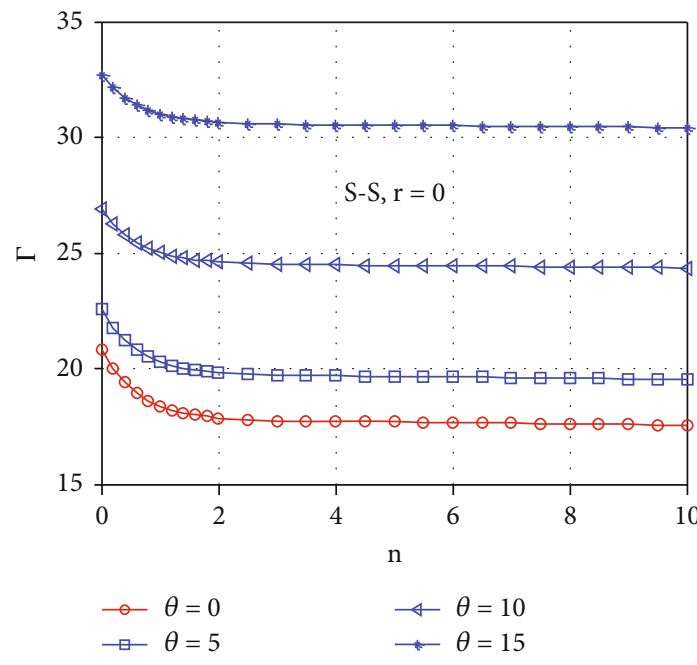

(a) S-S

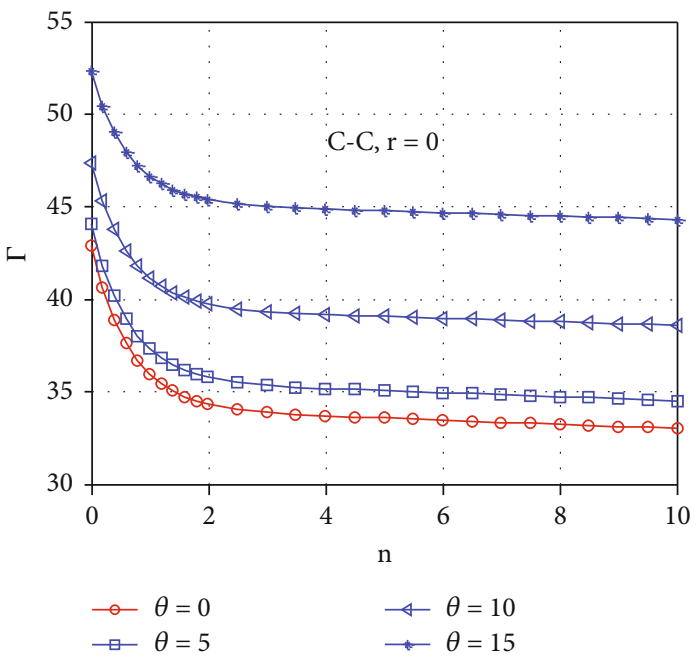

(b) C-C

FIGURE 2: The subordination of the first nondimensional fundamental frequencies of piezoelectric FG beam on the rotational speed and volume fraction index $n, K_{w}^{*}=10$, and $K_{s}^{*}=2$.

Example 3. Finally, the rotating beam's nondimensional fundamental frequencies as a function of speed are examined. In this computation, a cantilever structure with length $L$, depth $h=b=L / 100, r=0$, mass density $=2700 \mathrm{~kg} / \mathrm{m}^{3}$, and $E=70 \mathrm{GPa}$ is used. The frequency is normalized as follows:

$$
\wp_{i}=\omega_{i} L^{2} \sqrt{\frac{12 \rho}{E h^{2}}} .
$$

The first four nondimensional fundamental frequencies of the structure with several speeds are listed in Table 3. They were found using the exact solution [61], the novel dynamic modeling method (DMM) [62], and the isogeometric analysis [22].

\section{Numerical Studies and Comments about the Rotating Piezoelectric FG Beam}

The numerical investigations on natural vibration behaviors of rotating piezoelectric FG beams resting on Pasternak's elastic medium are carried out. Let us consider a beam structure with length $L$, depth $h$, and the same material characteristics for the FG core layer and the piezoelectric components as in Example 1. 


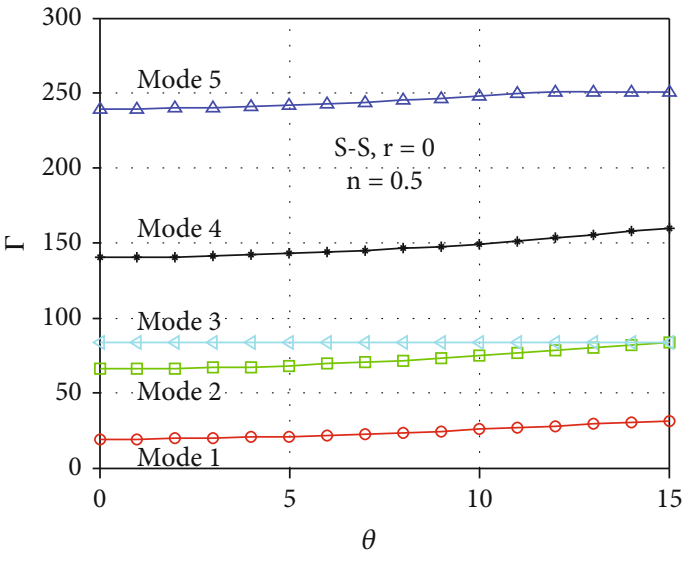

(a) S-S

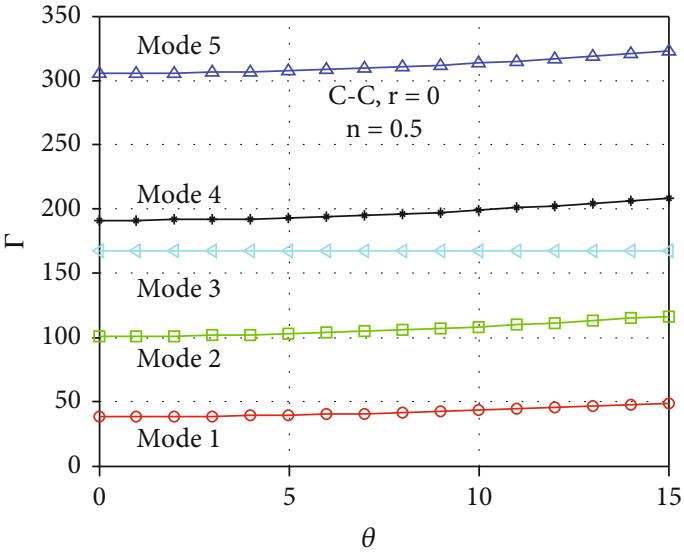

(b) C-C

FIGURE 3: The subordination of the first five nondimensional frequencies of piezoelectric FG beam on the rotational speed, $n=0.5, K_{w}^{*}=10$, and $K_{s}^{*}=2$.

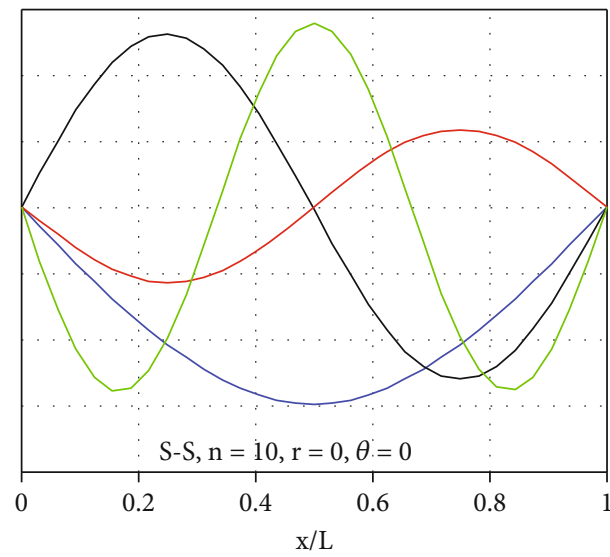

(a) $\theta=0$

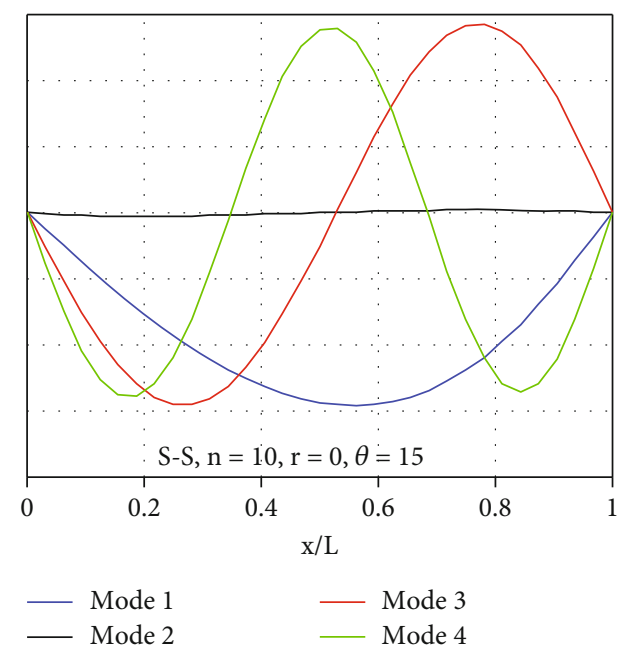

(b) $\theta=15$

FIGURE 4: The first four vibrational mode shapes of the rotating piezoelectric FG beam $(n=10, r=0)$.

The nondimensional natural frequency of the structure is normalized as follows:

$$
\Gamma=\omega L^{2} \sqrt{\frac{12 \rho_{0}}{E_{0} h^{2}}}
$$

in which, two Pasternak's elastic medium parameters $K_{w}^{*}$ and $K_{s}^{*}$ and the nondimensional rotational speed $\theta$ have the following expressions:

$$
K_{w}^{*}=\frac{k_{w} L^{4}}{D_{0}} ; K_{s}^{*}=\frac{k_{s} L^{2}}{\pi^{2} D_{0}} ; \theta=L^{2} \eta \sqrt{\frac{12 \rho_{0}}{E_{0} h^{2}}} ; D_{0}=\frac{E_{m} h^{3}}{12}
$$

4.1. Influence of Rotational Speed. To determine the impact of rotational speed on the natural vibration behavior of a rotating piezoelectric FG beam, vary the speed $\theta$ between 0 and 15 , while maintaining the following parameters: $L / h=$ $10, h_{p} / h_{a}=10, K_{w}^{*}=10, K_{s}^{*}=2$, and distance ratio $r / L=0$. Figure 2 illustrates the first nondimensional fundamental frequency as a consequence of the volume fraction index $n$ and the rotation speed. This finding demonstrates that the nondimensional natural frequency rises as the rotational speed increases. It is stated that when rotational speed rises, the structure's rigidity increases proportionately to centrifugal force. When the volume fraction index $n$ is increased, the percentage of metal in the structure rises. As the ratio of metal in the structure increases, both the stiffness and mass of the structure drop, with the mass decreasing more than the stiffness. Thus, the natural frequency of the structure drops, but the reduction is not progressive; the greatest fall in the natural frequency of the beam occurs when $n$ is changed from 0 to 2 . Additionally, this reduction is very dependent on the boundary conditions; the $\mathrm{C}-\mathrm{C}$ boundary 


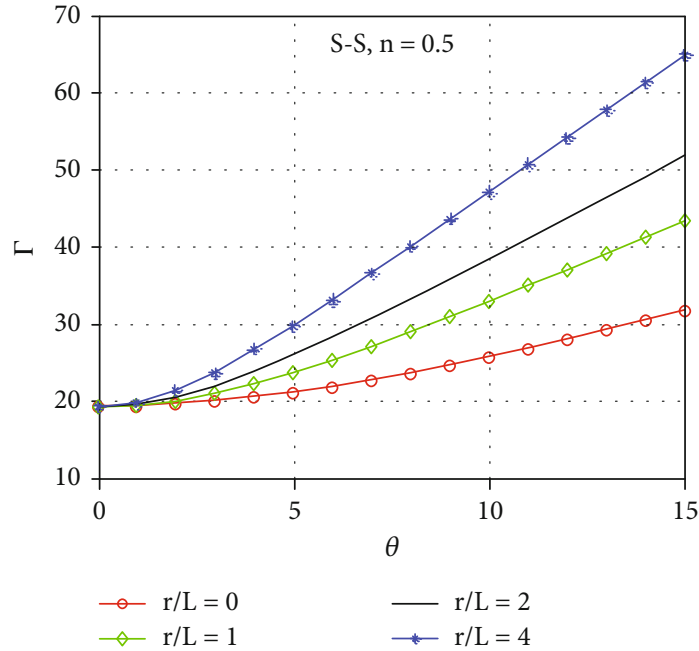

(a) S-S

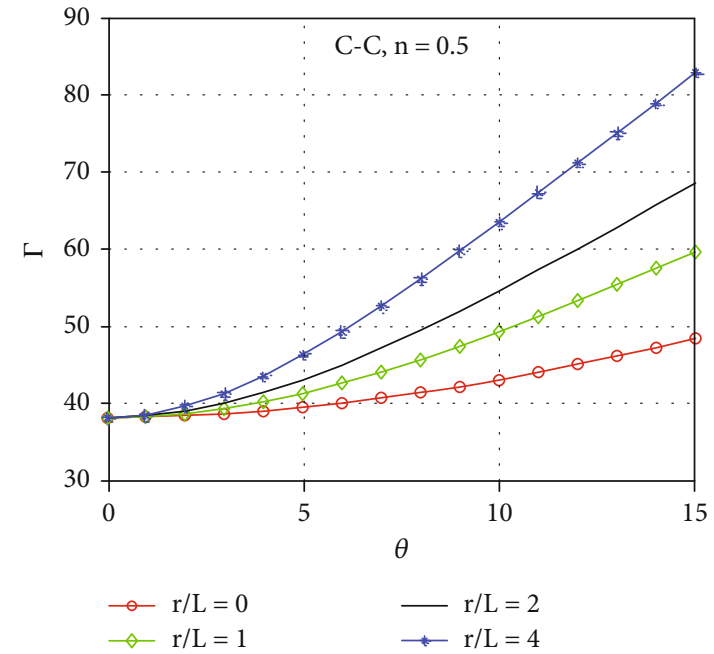

(b) C-C

FIGURE 5: The subordination of the first fundamental frequency of the piezoelectric FG beam on the ratio $r / L$ and on the rotating speed $(n=0.5)$.

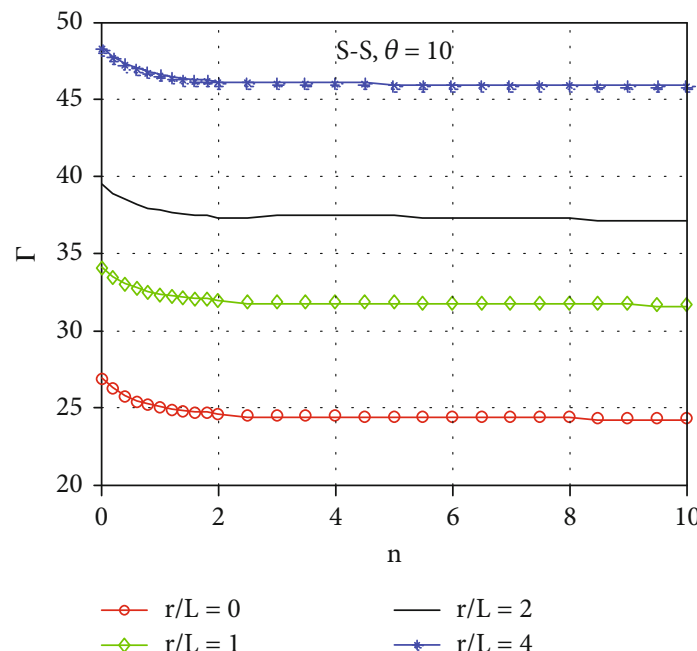

(a) S-S

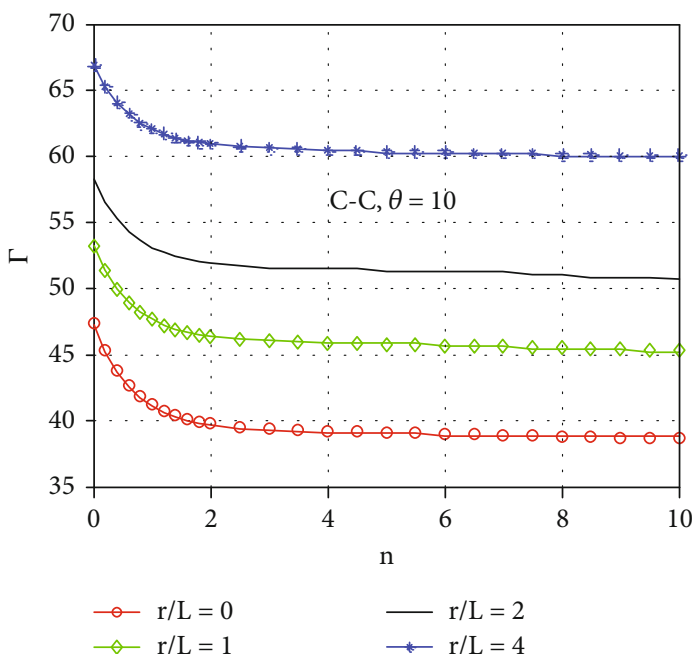

(b) $\mathrm{C}-\mathrm{C}$

Figure 6: The subordination of the first fundamental frequency of the piezoelectric FG beam on the ratio $r / L$ and $n(\theta=10)$.

exhibits a more pronounced drop than the S-S boundary, and when $n$ is larger than 2, both the C-C and S-S instances exhibit a small fall in natural frequency.

The influence of the piezoelectric beam's first five fundamental natural frequencies on the speed is shown in Figure 3 for the volume exponent $n=0.5$. As the rotational speed increases, the degree of expression of the rise in various free vibrations does not remain constant, with the third natural frequency slightly raised and the third natural frequency's dependent line nearly horizontal.

Figure 4 presents the first four vibrational mode shapes of the piezoelectric FG structure with speeds of 0 and 15; it can be observed easily that the rotational speed has a significant influence on the vibration shapes of the structure. As the speed of rotation is 0 , the first natural vibration of the beam is symmetrical through the midposition of the beam $(x=L / 2)$; however, for the case of speed $\theta=15$, the vibrational mode shapes of the structure are all deflected to the right side of the beam, that is, away from the axis of rotation. This may be explained by the fact that as the beam rotates at the rotational speed $\theta$, the geometry of the vibration mode is not any clearly symmetric all along the $x=L / 2$ axis and tends to move right of the beam's center. It is noteworthy that the structure does not exist without axial rotation.

4.2. Effect of the r/L Ratio. This section evaluates the effect of the distance $r$ between the beam's endpoint and the fixed 


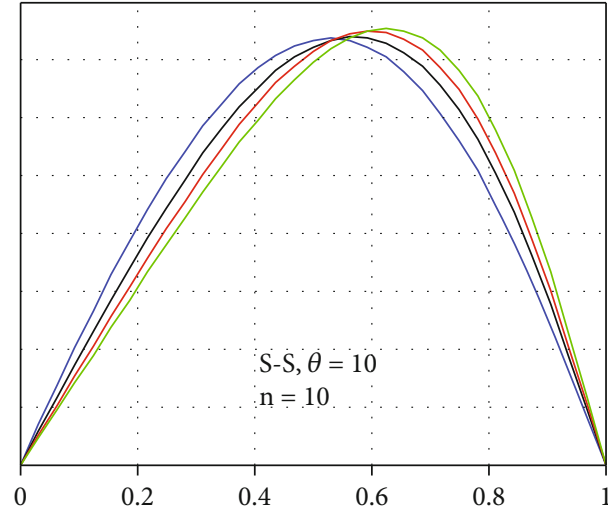

$\mathrm{x} / \mathrm{L}$

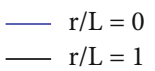

$\begin{aligned} \mathrm{r} / \mathrm{L} & =2 \\ \mathrm{r} / \mathrm{L} & =4\end{aligned}$

(a) S-S

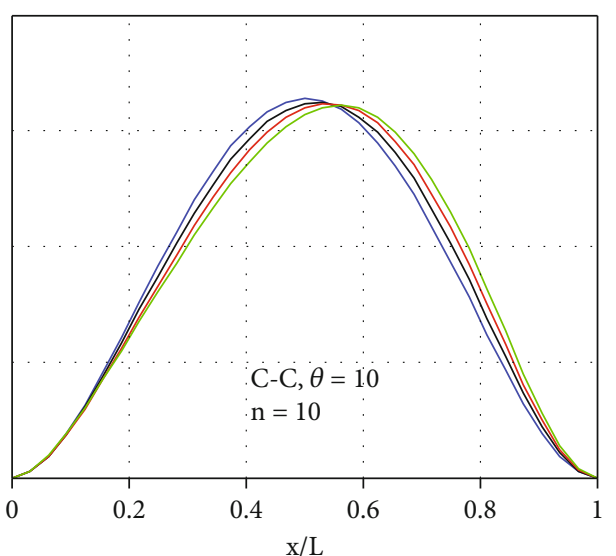

$-\mathrm{r} / \mathrm{L}=0$

$-\mathrm{r} / \mathrm{L}=2$

$\mathrm{r} / \mathrm{L}=4$

(b) $\mathrm{C}-\mathrm{C}$

Figure 7: The first four vibrational shapes of the rotating piezoelectric FG beam $(n=10, r=0)$.

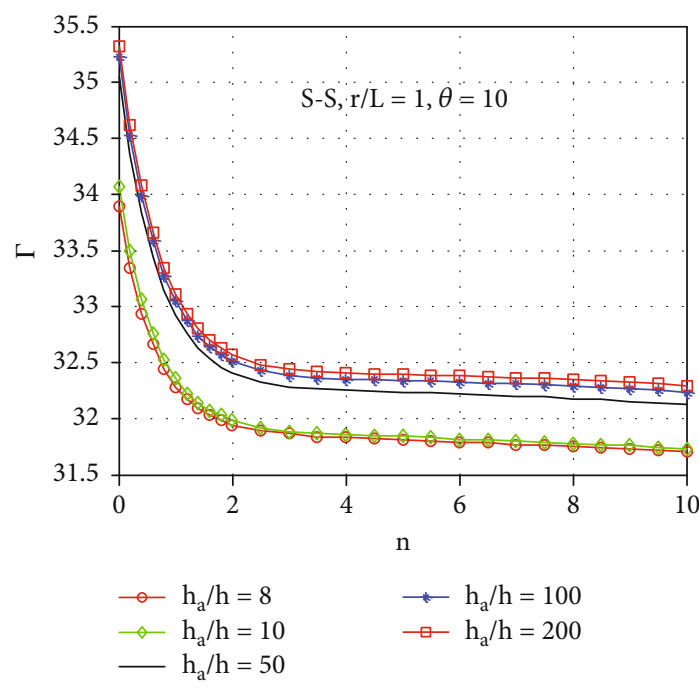

(a) S-S

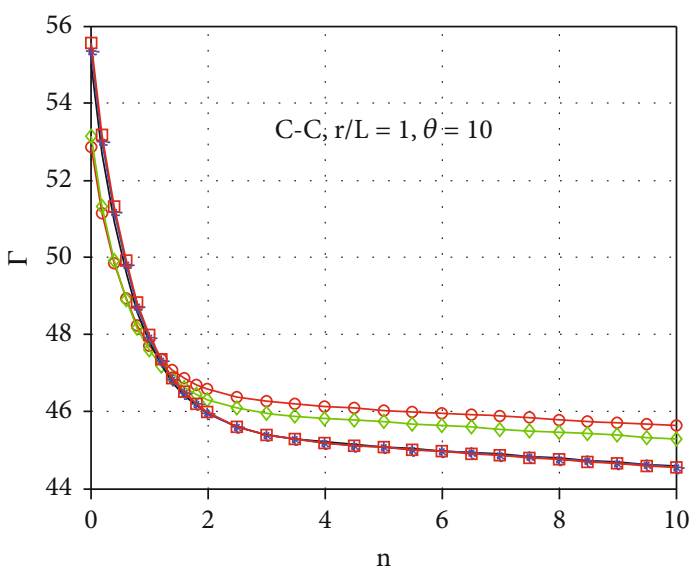

$\begin{aligned} \multimap & \mathrm{h}_{\mathrm{a}} / \mathrm{h}=8 \\ \smile \mathrm{h}_{\mathrm{a}} / \mathrm{h} & =10 \\ -\mathrm{h}_{\mathrm{a}} / \mathrm{h} & =50\end{aligned}$

$\rightarrow \mathrm{h}_{\mathrm{a}} / \mathrm{h}=100$

$\square \mathrm{h}_{\mathrm{a}} / \mathrm{h}=200$

(b) C-C

FIGURE 8: The subordination of the first frequencies of the piezoelectric FG structure on the ratio $h_{a} / h$ and $n, r / L=1$, and $\theta=10$.

axis; this distance value is given incrementally from 0 to $4 L$; Figure 5 illustrates the subordination of the first four fundamental frequencies on the $r / L$ ratio at various rotational speeds, Figure 6 illustrates the subordination of the first four fundamental frequencies on the $r / L$ ratio and the volume fraction proportion, and Figure 7 illustrates the first four vibrational modes. The numerical findings indicate the following:

(i) As the distance $r$ rises, the centrifugal force associated with the distance $r$ increases as well, increasing the stiffness of the piezoelectric FG structure and, thus, increasing the frequency of the beam

(ii) For the small values of the rotational speed $\theta(\theta<5)$, when the distance $r$ increases, the fundamental frequency of the structure increases slightly.
Nevertheless, for higher values of the rotational speed $\theta(\theta>5)$, this distance $r$ greatly affects the natural frequency of this beam

(iii) Figure 6 shows that for all the values of the volume fraction proportion $n$, the subordination of the first fundamental frequency on $n$ with four values of the distance $r$ is nearly identical and parallel to each other. This implies that when $r$ rises, the fundamental frequency of the structure increases proportionately and is independent of the material composition proportions. As for structure subjected to the C-C boundary, this only happens when the volume exponent $n>2$

(iv) As shown in Figure 7, when $r$ rises, the first vibrational mode of the structure's vibration changes and tends to move to the right. This demonstrates that $r$ influences not only the magnitude of the 

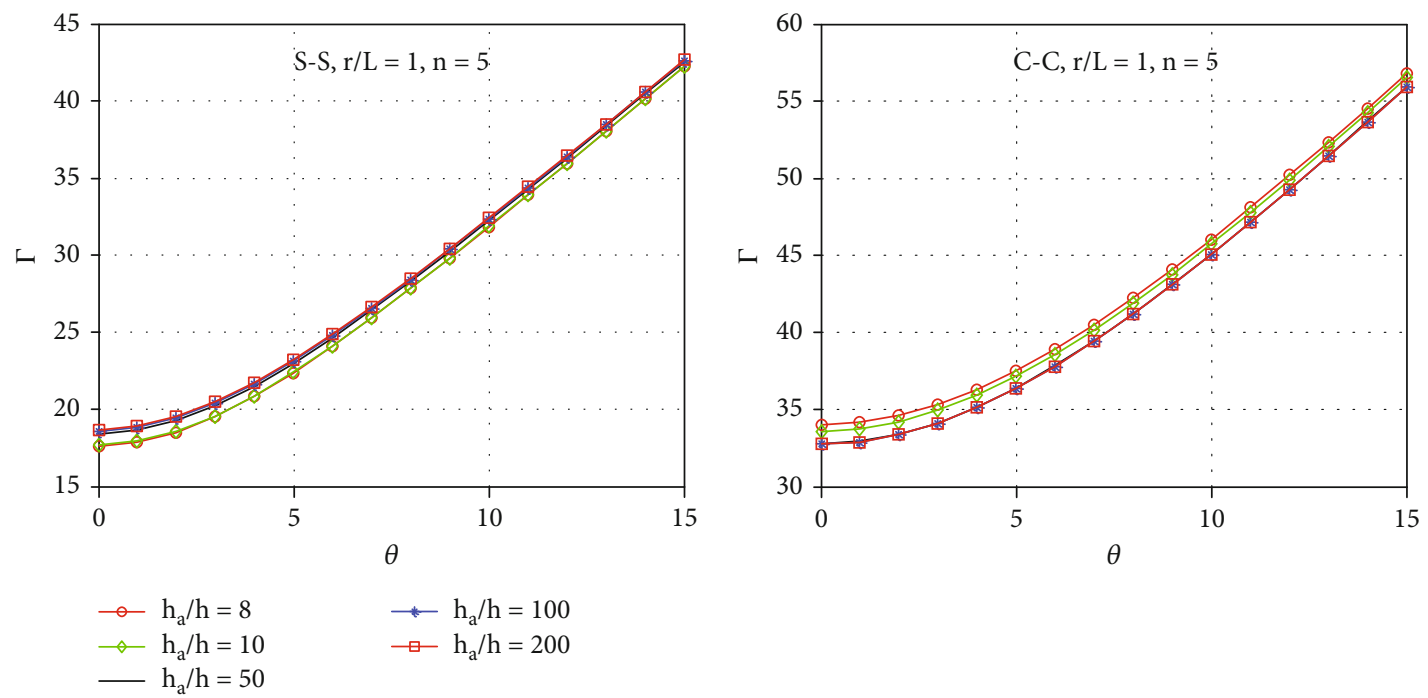

FIgURE 9: The subordination of the first frequencies of the piezoelectric FG structure on the ratio $h_{a} / h$ and $\theta, r / L=1$, and $n=5$.

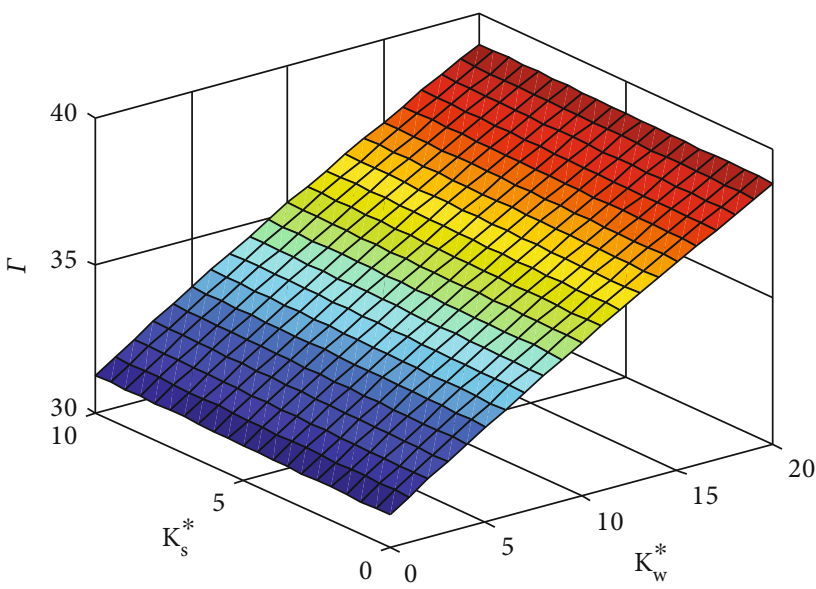

$\mathrm{S}-\mathrm{S}, \mathrm{r} / \mathrm{L}=1$

$\theta=10$

$\mathrm{n}=0.5$

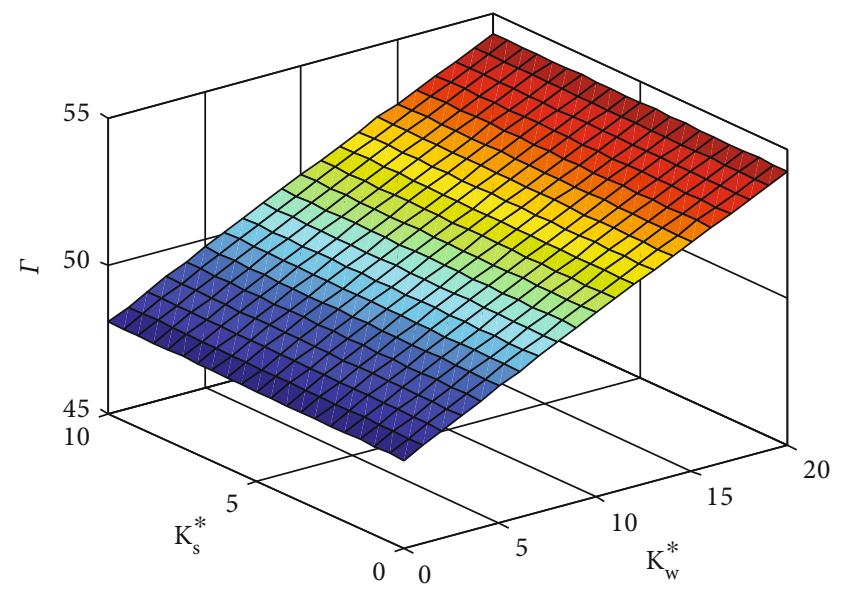

$\mathrm{C}-\mathrm{C}, \mathrm{r} / \mathrm{L}=1$

$\theta=10$

$\mathrm{n}=0.5$

(a) S-S

(b) $\mathrm{C}-\mathrm{C}$

FIgURE 10: The subordination of the first fundamental frequencies of the piezoelectric FG beam on $K_{w}^{*}$ and $K_{s}^{*}$ and $h / h_{a}=10$.

natural frequency but also the form of the piezoelectric FG beam's vibration mode

4.3. Influence of the Thickness of the Piezoelectric Layer $h_{a}$. Next, this section examines the effect of the thickness of the piezoelectric layer on the vibration behavior of the structure. A piezoelectric FG structure with the ratio $L / h$ $=10$ is unchanged (i.e., the thickness of the FG core layer does not change), $h_{a}$ is changed so that $h / h_{a}$ gets the values from 8 to $200, K_{w}^{*}=10$, and $K_{s}^{*}=2$. The subordination of the first natural frequency on the $h / h_{a}$ ratio and the volume fraction index is presented in Figure 8;
Figure 9 presents the subordination of the first fundamental frequency on the $h / h_{a}$ ratio and the rotating speed. One can see the following:

(i) For the S-S boundary, when the depth of the piezoelectric layer $h_{a}$ is decreased, the fundamental frequency of the piezoelectric FG structure increases

(ii) For the C-C boundary, when the volume fraction index $n$ increases gradually from 0 to around 1.2-1.4, the decrease in the thickness $h_{a}$ enhances the fundamental frequency of the structure. When $n>1.4$, the decrease 
in the thickness $h_{a}$ reduces the fundamental frequency of the structure. This indicates that there is a suitable value for the volume exponent $n$ and that the fundamental frequency of the piezoelectric FG structure varies very minimally as a function of the depth of the piezoelectric component; i.e., the natural frequency of this beam depends less on the value of the thickness of the piezoelectric layer. This also proves that the boundary constraints and the thickness of the piezoelectricity component both affect the vibration response of this beam

4.4. Influence of Elastic Foundation. Finally, the impact of the elastic medium on the vibrational behavior of the piezoelectric FG structure is explored in this study. Let us consider a beam with the $L / h$ ratio of $10, h / h a$ ratio of $10, r / L$ ratio of 1 , and rotating speed $\theta$ of 10 . Two elastic foundation parameters are modified to progressively rise from 0 to 20 , and $K_{s}^{*}$ gets the values in a range of 0 to 10 . The dependence of the beam's first natural frequency is seen in Figure 10. As can be seen, raising the values of two elastic medium parameters raises the piezoelectric FG beam's inherent frequency. As the elastic medium parameters increase, the energy of the structure increases accordingly and the overall stiffness of the piezoelectric FG structure increases, but the mass of the structure remains unchanged; the frequency of the structure increases.

\section{Conclusions}

This work combines the finite element method and the third-order shear deformation theory of Reddy to simulate the free vibration responses of the rotating piezoelectric FG structure resting on the two-parameter elastic medium. The numerical investigations show numerous novel phenomena, and some remarkable points can be pointed out as follows:

(i) As the rotational speed increases, the centrifugal force becomes greater and the fundamental frequency of the piezoelectric FG beam rises. Besides, the vibrational mode shapes of the structure change impressively as the speed rises; they tend to deviate significantly to the right side of the $L / 2$ position

(ii) As the distance from the endpoint of the beam to the rotation axis of the beam $r$ is increased, the fundamental frequency of the beam increases due to the increase in the whole stiffness of the beam

(iii) When the depth of the piezoelectric component is reduced, the boundary conditions significantly affect the fundamental frequency response of the piezoelectric FG beam. For the S-S case, the fundamental frequency increases as the thickness of the piezoelectric layer decreases. However, under the $\mathrm{C}-\mathrm{C}$ case, there will exist a value of volume exponent $n$ whose natural frequency is almost constant with different values of the depth of the piezoelectric layer

(iv) As the values of the elastic foundation become greater, the natural frequency of the beams increases

The above computed results show that the vibration response of the piezoelectric beam rotating around one fixed axis is different from that of the beam without rotation phenomenon because of the centrifugal inertia force generated by the rotational motion. This is a scientific basis for the design and use of beams with rotational motion in practice, taking into account the sensitivity of the speed parameter to the behavior of the rotating beam.

\section{Appendix}

The element stiffness matrix and element mass matrix as shown in equation (25) are written specifically as follows:

$$
\begin{aligned}
& \mathbf{K}_{e}^{\psi}=\int_{S_{e}}\left\{\sum_{i} \int_{h_{i}}^{h_{i+1}}\left(\begin{array}{c}
\mathbf{B}_{0}^{T} E(z) \mathbf{B}_{0}+\mathbf{B}_{0}^{T} E(z) z \mathbf{B}_{1}+\mathbf{B}_{0}^{T} E(z) z^{3} \mathbf{B}_{3} \\
+\mathbf{B}_{1}^{T} E(z) z \mathbf{B}_{0}+\mathbf{B}_{1}^{T} E(z) z^{2} \mathbf{B}_{1}+\mathbf{B}_{1}^{T} E(z) z^{4} \mathbf{B}_{3} \\
+\mathbf{B}_{3}^{T} E(z) z^{3} \mathbf{B}_{0}+\mathbf{B}_{3}^{T} E(z) z^{4} \mathbf{B}_{1}+\mathbf{B}_{3}^{T} E(z) z^{6} \mathbf{B}_{3} \\
+\mathbf{B}_{0 \gamma}^{T}\left(\frac{E(z)}{2(1+v(z))}\right) \mathbf{B}_{0 \gamma}+\mathbf{B}_{0 \gamma}^{T}\left(\frac{E(z) z^{2}}{2(1+v(z))}\right) \mathbf{B}_{2 \gamma} \\
+\mathbf{B}_{2 \gamma}^{T}\left(\frac{E(z) z^{2}}{2(1+v(z))}\right) \mathbf{B}_{0 \gamma}+\mathbf{B}_{2 \gamma}^{T}\left(\frac{E(z) z^{4}}{2(1+v(z))}\right) \mathbf{B}_{2 \gamma} \\
+k_{w} \mathbf{H}_{w}^{T} \mathbf{H}_{w}+k_{s}\left(\mathbf{H}_{w 1 x}{ }^{T} \mathbf{H}_{w 1 x}\right)
\end{array}\right\} d z\right. \\
& +\int_{S_{e}}\left\{\sum_{i} \int_{h_{i}}^{h_{i+1}}\left(\begin{array}{c}
\mathbf{B}_{0}^{T} E_{p} \mathbf{B}_{0}+\mathbf{B}_{0}^{T} E_{p} z \mathbf{B}_{1}+\mathbf{B}_{0}^{T} E_{p} z^{3} \mathbf{B}_{3}+\mathbf{B}_{1}^{T} E_{p} z \mathbf{B}_{0}+\mathbf{B}_{1}^{T} E_{p} z^{2} \mathbf{B}_{1} \\
+\mathbf{B}_{1}^{T} E_{p} z^{4} \mathbf{B}_{3}+\mathbf{B}_{3}^{T} E_{p} z^{3} \mathbf{B}_{0}+\mathbf{B}_{3}^{T} E_{p} z^{4} \mathbf{B}_{1}+\mathbf{B}_{3}^{T} E_{p} z^{6} \mathbf{B}_{3}
\end{array}\right) d z\right\} d S \\
& +\int_{S_{e}}\left\{\sum_{i} \int_{h_{i}}^{h_{i+1}}\left(\begin{array}{c}
\mathbf{B}_{0 \gamma}^{T}\left(G_{p}\right) \mathbf{B}_{0 \gamma}+\mathbf{B}_{0 \gamma}^{T}\left(G_{p} z^{2}\right) \mathbf{B}_{2 \gamma} \\
+\mathbf{B}_{2 \gamma}^{T}\left(G_{p} z^{2}\right) \mathbf{B}_{0 \gamma}+\mathbf{B}_{2 \gamma}^{T}\left(G_{p} z^{4}\right) \mathbf{B}_{2 \gamma}
\end{array}\right) d z\right\} d S, \\
& \mathbf{K}_{e}^{\psi \phi_{a}}=-\frac{1}{2} \int_{S_{e}}\left(-\mathbf{B}_{0}^{T} e_{31} B_{\phi}^{a}-\mathbf{B}_{1}^{T} z e_{31} B_{\phi}^{a}-\mathbf{B}_{3}^{T} z^{3} e_{31} B_{\phi}^{a}\right) d S, \\
& \mathbf{K}_{e}^{\psi \phi_{s}}=-\int_{S_{e}}\left(-\mathbf{B}_{0}^{T} e_{31} B_{\phi}^{s}-\mathbf{B}_{1}^{T} z e_{31} B_{\phi}^{s}-\mathbf{B}_{3}^{T} z^{3} e_{31} B_{\phi}^{s}\right) d S, \\
& \mathbf{K}_{e}^{\phi_{\alpha} \psi}=-\int_{S_{e}}\left(-\left(B_{\phi}^{a}\right)^{T} e_{31} \mathbf{B}_{0}-\left(B_{\phi}^{a}\right)^{T} e_{31} z \mathbf{B}_{1}-\left(B_{\phi}^{a}\right)^{T} e_{31} z^{3} \mathbf{B}_{3}\right) d S, \\
& \mathbf{K}_{e}^{\phi_{e} \psi}=-\int_{S_{c}}\left(-\left(B_{\phi}^{s}\right)^{T} e_{31} \mathbf{B}_{0}-\left(B_{\phi}^{s}\right)^{T} e_{31} z \mathbf{B}_{1}-\left(B_{\phi}^{s}\right)^{T} e_{31} z^{3} \mathbf{B}_{3}\right) d S, \\
& \mathbf{K}_{e}^{\phi_{\phi} \phi_{a}}=-\int_{S_{e}} B_{\phi}^{a} p_{33} B_{\phi} d S ; \mathbf{K}_{e}^{\phi_{s} \phi_{s}}=\int_{S_{e}} B_{\phi}^{s} p_{33} B_{\phi}^{s} d S .
\end{aligned}
$$

The following is the element stiffness matrix produced by rotation:

$$
\mathbf{K}_{e}^{R}=\int_{L}\left(Q_{\phi}(x) \mathbf{H}_{w 1 x}^{T} \mathbf{H}_{w 1 x}\right) d x .
$$

The following is the element stiffness matrix:

$$
\mathbf{M}_{e}=\int \sum_{S} \int_{i} \int_{h_{i}}^{h_{i+1}}\left(\rho(z)+\rho_{p}\right)\left(\mathbf{H}_{\psi}{ }^{T} \mathbf{H}_{\psi}\right) d z d S,
$$

where $d S=d x d y$; the calculation of these matrices uses the Gaussian integration as in the document [63], and the 
coordinates of any point with the coordinate $(x, y)$ in the element being calculated in the Cartesian coordinate system $(x, y)$ need to be converted to the natural coordinate system as follows $(\xi, \varsigma)$ :

$$
\left\{\begin{array}{l}
x=\sum_{i=1}^{2} N_{i} x_{i}, \\
y=\sum_{i=1}^{2} N_{i} y_{i},
\end{array}\right.
$$

where $N_{i}$ are shape functions calculated in terms of the natural coordinates system $(\xi, \varsigma)$ and $-1 \leq \xi$, $\varsigma \leq 1$..

The Jacobian matrix from the coordinate system transformation [59] is

$$
\mathbf{J}=\left[\begin{array}{ll}
\frac{\partial x}{\partial \xi} & \frac{\partial y}{\partial \xi} \\
\frac{\partial x}{\partial \varsigma} & \frac{\partial y}{\partial \varsigma}
\end{array}\right]
$$

At this time, the element stiffness and the element mass matrices are calculated as follows:

$$
\begin{aligned}
& \mathbf{K}_{e}^{\psi}=\int_{-1}^{1} \int_{-1}^{1}\left\{\sum_{i} \int_{h_{i}}^{h_{i+1}}\left(\begin{array}{c} 
\\
\mathbf{B}_{0}^{T} E(z) \mathbf{B}_{0}+\mathbf{B}_{0}^{T} E(z) z \mathbf{B}_{1}+\mathbf{B}_{0}^{T} E(z) z^{3} \mathbf{B}_{3} \\
+\mathbf{B}_{1}^{T} E(z) z \mathbf{B}_{0}+\mathbf{B}_{1}^{T} E(z) z^{2} \mathbf{B}_{1}+\mathbf{B}_{1}^{T} E(z) z^{4} \mathbf{B}_{3} \\
+\mathbf{B}_{3}^{T} E(z) z^{3} \mathbf{B}_{0}+\mathbf{B}_{3}^{T} E(z) z^{4} \mathbf{B}_{1}+\mathbf{B}_{3}^{T} E(z) z^{6} \mathbf{B}_{3} \\
+\mathbf{B}_{0 \gamma}^{T}\left(\frac{E(z)}{2(1+v(z))}\right) \mathbf{B}_{0 \gamma}+\mathbf{B}_{0 \gamma}^{T}\left(\frac{E(z) z^{2}}{2(1+v(z))}\right) \mathbf{B}_{2 \gamma} \\
+\mathbf{B}_{2 \gamma}^{T}\left(\frac{E(z) z^{2}}{2(1+v(z))}\right) \mathbf{B}_{0 \gamma}+\mathbf{B}_{2 \gamma}^{T}\left(\frac{E(z) z^{4}}{2(1+v(z))}\right) \mathbf{B}_{2 \gamma} \\
+k_{w} \mathbf{H}_{w}^{T} \mathbf{H}_{w}+k_{s}\left(\mathbf{H}_{w 1 x}{ }^{T} \mathbf{H}_{w 1 x}\right)
\end{array}\right\} d z\right\} d \xi d \varsigma \\
& +\int_{-1}^{1} \int_{-1}^{1}\left\{\sum_{i} \int_{h_{i}}^{h_{i+1}}\left(\begin{array}{c}
\mathbf{B}_{0}^{T} E_{p} \mathbf{B}_{0}+\mathbf{B}_{0}^{T} E_{p} z \mathbf{B}_{1}+\mathbf{B}_{0}^{T} E_{p} z^{3} \mathbf{B}_{3}+\mathbf{B}_{1}^{T} E_{p} z \mathbf{B}_{0}+\mathbf{B}_{1}^{T} E_{p} z^{2} \mathbf{B}_{1} \\
+\mathbf{B}_{1}^{T} E_{p} z^{4} \mathbf{B}_{3}+\mathbf{B}_{3}^{T} E_{p} z^{3} \mathbf{B}_{0}+\mathbf{B}_{3}^{T} E_{p} z^{4} \mathbf{B}_{1}+\mathbf{B}_{3}^{T} E_{p} z^{6} \mathbf{B}_{3}
\end{array}\right) d z\right\} d \xi d \varsigma \\
& +\int_{-1}^{1} \int_{-1}^{1}\left\{\sum_{i} \int_{h_{i}}^{h_{i+1}}\left(\begin{array}{c}
\mathbf{B}_{0 \gamma}^{T}\left(G_{p}\right) \mathbf{B}_{0 \gamma}+\mathbf{B}_{0 \gamma}^{T}\left(G_{p} z^{2}\right) \mathbf{B}_{2 \gamma} \\
+\mathbf{B}_{2 \gamma}^{T}\left(G_{p} z^{2}\right) \mathbf{B}_{0 \gamma}+\mathbf{B}_{2 \gamma}^{T}\left(G_{p} z^{4}\right) \mathbf{B}_{2 \gamma}
\end{array}\right) d z\right\} J d \xi d \varsigma, \\
& \mathbf{K}_{e}^{\psi \phi_{a}}=-\int_{-1}^{1} \int_{-1}^{1}\left(-\mathbf{B}_{0}^{T} e_{31} B_{\phi}^{a}-\mathbf{B}_{1}^{T} z e_{31} B_{\phi}^{a}-\mathbf{B}_{3}^{T} z^{3} e_{31} B_{\phi}^{a}\right) J d \xi d \zeta, \\
& \mathbf{K}_{e}^{\psi \phi_{s}}=-\int_{-1}^{1} \int_{-1}^{1}\left(-\mathbf{B}_{0}^{T} e_{31} B_{\phi}^{s}-\mathbf{B}_{1}^{T} z e_{31} B_{\phi}^{s}-\mathbf{B}_{3}^{T} z^{3} e_{31} B_{\phi}^{s}\right) J d \xi d \varsigma, \\
& \mathbf{K}_{e}^{\phi_{e} \psi}=-\int_{-1}^{1} \int_{-1}^{1}\left(-\left(B_{\phi}^{a}\right)^{T} e_{31} \mathbf{B}_{0}-\left(B_{\phi}^{a}\right)^{T} e_{31} z \mathbf{B}_{1}-\left(B_{\phi}^{a}\right)^{T} e_{31} z^{3} \mathbf{B}_{3}\right) J d \xi d \varsigma, \\
& \mathbf{K}_{e}^{\phi, \psi}=-\int_{-1}^{1} \int_{-1}^{1}\left(-\left(B_{\phi}^{s}\right)^{T} e_{31} \mathbf{B}_{0}-\left(B_{\phi}^{s}\right)^{T} e_{31} z \mathbf{B}_{1}-\left(B_{\phi}^{s}\right)^{T} e_{31} z^{3} \mathbf{B}_{3}\right) J d \xi d \varsigma, \\
& \mathbf{K}_{e}^{\phi_{a} \phi_{a}}=-\int_{-1}^{1} \int_{-1}^{1} B_{\phi}^{a} p_{33} B_{\phi} J d \xi d \zeta ; \mathbf{K}_{e}^{\phi_{s} \phi_{s}}=\int_{-1}^{1} \int_{-1}^{1} B_{\phi}^{s} p_{33} B_{\phi}^{s} J d \xi d \zeta, \\
& \mathbf{K}_{e}^{R}=\int_{-1}^{1}\left(F_{\eta}(\varsigma) \mathbf{H}_{w 1 x}^{T} \mathbf{H}_{w 1 x}\right) J d \xi, \\
& \mathbf{M}_{e}=\int_{-1}^{1} \int_{-1}^{1} \sum_{i} \int_{h_{i}}^{h_{i+1}}\left(\rho(z)+\rho_{p}\right)\left(\mathbf{H}_{\psi}{ }^{T} \mathbf{H}_{\psi}\right) d z J d \xi d \zeta,
\end{aligned}
$$

with $J=\operatorname{det}(\mathbf{J})$.

\section{Data Availability}

Data used to support the findings of this study are included in the article.

\section{Conflicts of Interest}

All authors declare that there is no conflict of interest regarding the publication of this paper.

\section{Acknowledgments}

This work was supported by the University of Transport and Communications Foundation for Science and Technology Development (Grant number 786/TB-DHGTVT).

\section{References}

[1] J. N. Reddy, "Free vibration analysis of functionally graded ceramic-metal plates," Analysis and Design of Plated Structures, vol. 1, pp. 293-321, 2007.

[2] Z. Ning, K. Tahir, G. Haomin, S. Shaoshuai, Z. Wei, and Z. Weiwei, "Functionally graded materials: an overview of stability, buckling, and free vibration analysis," Advances in Materials Science and Engineering, vol. 2019, Article ID 1354150, 18 pages, 2019.

[3] D. V. Thom, D. H. Duc, P. V. Minh, and N. S. Tung, "Finite element modelling for free vibration response of cracked stiffened FGM plates," Vietnam Journal Of Science And Technology, vol. 58, pp. 119-129, 2020.

[4] P. V. Vinh, N. T. Dung, N. C. Tho, D. V. Thom, and L. K. Hoa, "Modified single variable shear deformation plate theory for free vibration analysis of rectangular FGM plates," Structure, vol. 29, pp. 1435-1444, 2021.

[5] H. N. Nguyen, T. C. Tan, D. T. Luat, V. D. Phan, D. V. Thom, and P. V. Minh, "Research on the buckling behavior of functionally graded plates with stiffeners based on the third-order shear deformation theory," Materials, vol. 12, no. 8, p. 1262, 2019.

[6] X. Zhao, S. Zheng, and Z. Li, "Effects of porosity and flexoelectricity on static bending and free vibration of AFG piezoelectric nanobeams," Thin-Walled Structures, vol. 151, 2020.

[7] Z. Nan, Z. Xie, Z. Shijie, and C. Dejin, "Size-dependent static bending and free vibration analysis of porous functionally graded piezoelectric nanobeams," Smart Materials and Structures, vol. 29, no. 4, article 045025, 2020.

[8] A. Singh and P. Kumari, "Two-dimensional free vibration analysis of axially functionally graded beams integrated with piezoelectric layers: an piezoelasticity approach," International Journal of Applied Mechanics, vol. 12, no. 4, 2020.

[9] K. Larkin, A. Hunter, and A. Abdelkefi, "Size-dependent modeling and performance enhancement of functionally graded piezoelectric energy harvesters," Journal of Nanoparticle Research, vol. 22, p. 121, 2020.

[10] M. Y. Yasin, B. Prakash, and A. H. Khan, "Finite element model based on an efficient layerwise theory for dynamics and active vibration control of smart functionally graded beams," Materials Research Express, vol. 7, no. 2, article 025703, 2020.

[11] Q. Chen, S. Zheng, Z. Li, and C. Zeng, "Size-dependent free vibration analysis of functionally graded porous piezoelectric sandwich nanobeam reinforced with graphene platelets with consideration of flexoelectric effect," Smart Materials and Structures, vol. 30, no. 3, article 035008, 2021.

[12] M. Askari, E. Brusa, and C. Delprete, "On the vibration analysis of coupled transverse and shear piezoelectric functionally graded porous beams with higher-order theories," The Journal 
of Strain Analysis for Engineering Design, vol. 56, no. 1, pp. 2949, 2021.

[13] S. K. Gunjal and U. S. Dixit, "Vibration analysis of shapeoptimized rotating cantilever beams," Engineering Optimization, vol. 39, no. 1, pp. 105-123, 2007.

[14] S. C. Pradhan and T. Murmu, "Application of nonlocal elasticity and DQM in the flapwise bending vibration of a rotating nanocantilever," Physica E: Physica E: Low-dimensional Systems and Nanostructures, vol. 42, pp. 1944-1949, 2010.

[15] L. Li, D. G. Zhang, and W. D. Zhu, "Free vibration analysis of a rotating hub-functionally graded material beam system with the dynamic stiffening effect," Journal of Sound and Vibration, vol. 333, pp. 1526-1541, 2014.

[16] M. D. S. Amir, B. J. Mehdi, and M. Dehrouyeh, "On sizedependent lead-lag vibration of rotating microcantilevers," International Journal of Engineering Science, vol. 101, pp. 5063, 2016.

[17] L. Jung-Woo and L. Jung-Youn, "In-plane bending vibration analysis of a rotating beam with multiple edge cracks by using the transfer matrix method," Meccanica, vol. 52, no. 4-5, pp. 1143-1157, 2017.

[18] D. Das, "Free vibration and buckling analyses of geometrically non-linear and sheardeformable FGM beam fixed to the inside of a rotating rim," Composite Structures, vol. 179, pp. 628-645, 2017.

[19] X. Xu, Q. Han, and F. Chu, "Vibration suppression of a rotating cantilever beam under magnetic excitations by applying the magnetostrictive material," Journal of Intelligent Material Systems and Structures, vol. 30, no. 4, pp. 576-592, 2019.

[20] B. Alireza and X. Y. Cai, "Vibration analysis of rotating rods based on the nonlocal elasticity theory and coupled displacement field," Microsystem Technologies, vol. 25, pp. 10771085, 2019.

[21] L. Liang, L. Wei-Hsin, Z. Dingguo, and Z. Yang, "Vibration control and analysis of a rotating flexible FGM beam with a lumped mass in temperature field," Composite Structures, vol. 208, pp. 244-260, 2019.

[22] C. Dejin, F. Kai, and Z. Shijie, "Flapwise vibration analysis of rotating composite laminated Timoshenko microbeams with geometric imperfection based on a re-modified couple stress theory and isogeometric analysis," European Journal of Mechanics - A/Solids, vol. 76, pp. 25-35, 2019.

[23] M. S. Atanasov and V. Stojanović, "Nonlocal forced vibrations of rotating cantilever nano-beams," European Journal of Mechanics - A/Solids, vol. 79, article ???, 2020.

[24] B. T. T. Mai, N. H. Cuong, N. D. Quang, and D. H. Tai, "Experimental study on flexural and shear behaviour of sandwich panels using glass textile reinforced concrete and autoclaved aerated concrete," Transport and Communications Science Journal, vol. 71, no. 1, pp. 18-26, 2020.

[25] S. Khosravi, H. Arvin, and Y. Kiani, "Vibration analysis of rotating composite beams reinforced with carbon nanotubes in thermal environment," International Journal of Mechanical Sciences, vol. 164, article 105187, 2019.

[26] S. Khosravi, H. Arvin, and Y. Kiani, "Interactive thermal and inertial buckling of rotating temperature-dependent FG-CNT reinforced composite beams," Composites Part B: Engineering, vol. 175, article 107178, 2019.

[27] Y. Kiani, H. Bagheri, and M. R. Eslami, "Asymmetric compressive stability of rotating annular plates," European Journal of Computational Mechanics, vol. 28, no. 4, pp. 325-350, 2019.
[28] H. Arvin, S. M. H. Hosseini, and Y. Kiani, "Free vibration analysis of pre/post buckled rotating functionally graded beams subjected to uniform temperature rise," Thin-Walled Structures, vol. 158, article 107187, 2021.

[29] S. M. H. Hosseini, H. Arvin, and Y. Kiani, "On buckling and post-buckling of rotating clamped-clamped functionally graded beams in thermal environment," Mechanics Based Design of Structures and Machines, pp. 1-16, 2020.

[30] H. Bagheri, Y. Kiani, and M. R. Eslami, "Asymmetric thermoinertial buckling of annular plates," Acta Mechanica, vol. 228, no. 4, pp. 1493-1509, 2017.

[31] M. Komijania, Y. Kiania, S. E. Esfahanib, and M. R. Eslami, "Vibration of thermo-electrically post-buckled rectangular functionally graded piezoelectric beams," Composite Structures, vol. 98, pp. 143-152, 2013.

[32] Y. Kiani, M. Rezaei, S. Taheri, and M. R. Eslami, “Thermoelectrical buckling of piezoelectric functionally graded material Timoshenko beams," International Journal of Mechanics and Materials in Design, vol. 7, pp. 185-197, 2011.

[33] Y. Kiani, S. Taheri, and M. R. Eslami, "Thermal buckling of piezoelectric functionally graded material beams," Journal of Thermal Stresses, vol. 34, no. 8, pp. 835-850, 2011.

[34] A. Kargani and E. M. R. Kiani, "Exact solution for nonlinear stability of piezoelectric FGM Timoshenko beams under thermo-electrical loads," Journal of Thermal Stresses, vol. 36, no. 10, pp. 1056-1076, 2013.

[35] M. Komijiani, Y. Kiani, and M. R. Eslami, "Non-linear thermoelectrical stability analysis of functionally graded piezoelectric material beams," Journal of Intelligent Material Systems and Structures, vol. 24, no. 4, pp. 399-410, 2013, Người đọc có thể tìm thấy thêm các kết quả đáp ứng cơ học của dầm áp điện FGM trong các công trình [42]-[46].

[36] G. Moustafa, S. Hayat, B. Fouad et al., "Influences of porosity distributions and boundary conditions on mechanical bending response of functionally graded plates resting on Pasternak foundation," Steel \& Composite Structures, vol. 38, no. 1, pp. 1-15, 2021.

[37] R. Abdelkrim, C. Abdelbaki, A. B. Abdelmoumen et al., "Physical stability response of a SLGS resting on viscoelastic medium using nonlocal integral first-order theory," Steel \& Composite Structures, vol. 37, no. 6, pp. 695-709, 2020.

[38] B. Noureddine, Z. Mohamed, A. B. Abdelmoumen et al., "Deflections, stresses and free vibration studies of FG-CNT reinforced sandwich plates resting on Pasternak elastic foundation," Computers and Concrete, vol. 26, no. 3, pp. 213-226, 2020.

[39] M. S. H. Al-Furjan, H. Mostafa, N. Jing, W. J. Dong, and T. Abdelouahed, "Frequency simulation of viscoelastic multiphase reinforced fully symmetric systems," Engineering with Computers, 2020, Published 27 October 2020.

[40] R. Mohamed, H. B. Kouider, K. Abdelhakim et al., "A new innovative 3-unknowns HSDT for buckling and free vibration of exponentially graded sandwich plates resting on elastic foundations under various boundary conditions," Geomechanics and Engineering, vol. 22, no. 2, pp. 119-132, 2020.

[41] C. C. Sara, K. Abdelhakim, A. B. Abdelmoumen et al., "A novel four-unknown integral model for buckling response of FG sandwich plates resting on elastic foundations under various boundary conditions using Galerkin's approach," Geomechanics and Engineering, vol. 21, no. 5, pp. 471-487, 2020. 
[42] R. Salah, A. B. Abdelmoumen, B. Abdelhakim et al., "Effects of hygro-thermo-mechanical conditions on the buckling of FG sandwich plates resting on elastic foundations," Computers and Concrete, vol. 25, no. 4, pp. 311-325, 2020.

[43] B. Moussa, H. Habib, H. Houari et al., "Buckling behavior of a single-layered graphene sheet resting on viscoelastic medium via nonlocal four-unknown integral model," Steel \& Composite Structures, vol. 34, no. 5, pp. 643-655, 2020.

[44] K. Miloud, K. Abdelhakim, A. B. Abdelmoumen et al., "Free vibration and static bending analysis of piezoelectric functionally graded material plates resting on one area of twoparameter elastic foundation," Computers and Concrete, vol. 2020, no. 1, pp. 1-18, 2020.

[45] T. Abdelouahed, S. U. Al-Dulaijan, M. A. Al-Osta et al., "A four variable trigonometric integral plate theory for hygrothermo-mechanical bending analysis of AFG ceramic-metal plates resting on a two-parameter elastic foundation," Steel \& Composite Structures, vol. 34, no. 4, pp. 511-524, 2020.

[46] Y. A. Farouk, M. Mustapha, A. B. Abdelmoumen et al., "Influences of porosity on dynamic response of FG plates resting on Winkler/Pasternak/Kerr foundation using quasi 3D HSDT," Computers and Concrete, vol. 24, no. 4, pp. 347-367, 2019.

[47] I. T. Saeed, C. Abdelbaki, T. Abdelouahed, M. A. Al-Ostaa, S. U. Al-Dulaijan, and M. M. Al-Zahrania, "Wave propagation analysis of a ceramic-metal functionally graded sandwich plate with different porosity distributions in a hygro-thermal environment," Composite Structures, vol. 269, article 114030, 2021.

[48] H. Houari, A. B. Abdelmoumen, K. Abdelhakim et al., "Bending analysis of functionally graded plates using a new refined quasi-3D shear deformation theory and the concept of the neutral surface position," Steel \& Composite Structures, vol. 39, no. 1, pp. 051-064, 2021.

[49] B. Ahmed, B. Fouad, A. B. Abdelmoumen et al., "Buckling analysis of functionally graded plates using HSDT in conjunction with the stress function method," Computers and Concrete, vol. 27, no. 1, pp. 73-83, 2021.

[50] Q. B. Tinh, V. D. Thom, H. T. T. Lan et al., "On the high temperature mechanical behaviors analysis of heated functionally graded plates using FEM and a new third-order shear deformation plate theory," Composites Part B: Engineering, vol. 92, pp. 218-241, 2016.

[51] T. Yu, T. Q. Bui, S. Yin et al., "On the thermal buckling analysis of functionally graded plates with internal defects using extended isogeometric analysis," Composite Structures, vol. 136, pp. 684-695, 2016.

[52] P. T. Dat, D. V. Thom, and D. T. Luat, "Free vibration of functionally graded sandwich plates with stiffeners based on the third-order shear deformation theory," Vietnam Journal of Mechanics, vol. 38, no. 2, pp. 103-122, 2016.

[53] N. D. Duc, T. D. Trinh, T. V. Do, and D. H. Doan, "On the buckling behavior of multi-cracked FGM plates," International Conference on Advances in Computational Mechanics, Springer, Singapore, 2018.

[54] V. T. Do, D. H. Doan, N. D. Duc, and T. Q. Bui, "Phase-field thermal buckling analysis for cracked functionally graded composite plates considering neutral surface," Composite Structures, vol. 182, pp. 542-548, 2017.

[55] P. M. Phuc, “Analysis free vibration of the functionally grade material cracked plates with varying thickness using the phase-field theory," Transport and Communications Science Journal, vol. 70, no. 2, article 1573776000, pp. 122-131, 2019.
[56] M. Y. Yasin, N. Ahmad, and M. N. Alam, "Finite element analysis of actively controlled smart plate with patched actuators and sensors," Latin American Journal of Solids and Structures, vol. 7, no. 3, pp. 227-247, 2010.

[57] S. Valliappan and K. Qi, "Finite element analysis of a smart damper for seismic structural control," Computers \& Structures, vol. 81, pp. 1009-1017, 2003.

[58] H. S. Tzou and C. I. Tseng, "Distributed piezoelectric sensor/ actuator design for dynamicmeasurement/control of distributed parameter systems: a piezoelectric finite elementapproach," Journal of Sound and Vibration, vol. 138, pp. 17-34, 1990.

[59] S. P. Timoshenko and J. M. Gere, Theory of Elastic Stability, Dover Publications, 1989.

[60] M. Avcar and K. M. M. Waleed, "Free vibration of functionally graded beams resting on Winkler-Pasternak foundation," Arabian Journal of Geosciences, vol. 11, no. 10, p. 232, 2018.

[61] A. D. Wright, C. E. Smith, R. W. Thresher, and J. L. C. Wang, "Vibration modes of centrifugaily stiffened beams," Journal of Applied Mechanics, vol. 49, pp. 197-202, 1982.

[62] H. H. Yoo and S. H. Shin, "Vibration analysis of rotating cantilever beams," Journal of Sound and Vibration, vol. 212, no. 5, pp. 807-828, 1998.

[63] J. N. Reddy, An Introduction to the Finite Element Method, McGraw-Hill, New York, 2005. 